J. DIFFERENTIAL GEOMETRY

88 (2011) 333-377

\title{
SEIBERG-WITTEN EQUATIONS, END-PERIODIC DIRAC OPERATORS, AND A LIFT OF ROHLIN'S INVARIANT
}

\author{
Tomasz Mrowka, Daniel Ruberman \& Nikolai Saveliev
}

\begin{abstract}
We introduce a gauge-theoretic integer valued lift of the Rohlin invariant of a smooth 4-manifold $X$ with the homology of $S^{1} \times S^{3}$. The invariant has two terms: one is a count of solutions to the Seiberg-Witten equations on $X$, and the other is essentially the index of the Dirac operator on a non-compact manifold with end modeled on the infinite cyclic cover of $X$. Each term is metric (and perturbation) dependent, and we show that these dependencies cancel as the metric and perturbation vary in a generic 1-parameter family.
\end{abstract}

\section{Introduction}

We use the Seiberg-Witten equations to define an integer valued invariant of an oriented smooth closed 4-manifold $X$ with the integral homology of $S^{1} \times S^{3}$ that reduces mod 2 to the Rohlin invariant $[42,43,48]$

of $X$. Manifolds with this homology type arise, for instance, from furling up a homology cobordism from an integral homology sphere to itself; hence their study should shed light on classical problems concerning the homology cobordism group $\Theta_{3}^{H}$ of integral homology spheres and the classical Rohlin invariant $\rho: \Theta_{3}^{H} \rightarrow \mathbb{Z} / 2$. In addition, the basic example of such a manifold, namely $S^{1} \times S^{3}$ itself, is perhaps the simplest smooth 4-manifold that might admit an exotic smooth structure, detectable in principle by the Rohlin invariant (the Kirby-Siebenmann invariant $[\mathbf{2 2}]$ in this dimension).

Because $b_{+}^{2}(X)=0$, the usual count of solutions to the SeibergWitten equations on $X$ will depend on choices of metric and perturbation. The main work in the paper is to define and understand a correction term to this count, based on a study of the spin Dirac operator on a non-compact manifold with end modeled on the infinite cyclic cover of $X$. The index of this operator is defined using Taubes' theory $[\mathbf{5 1}]$ of periodic-end operators; we extend this theory by proving a

Received 9/1/2009. 
change of index formula for 1-parameter families of such operators in terms of version of spectral flow.

In summary, the invariant is defined as follows. The Seiberg-Witten equations on $X$ depend on two parameters: a Riemannian metric $g$ and a perturbing self-dual 2 -form, written as $d^{+} \beta$. For generic choices of these parameters, the Seiberg-Witten moduli space $\mathcal{M}(X, g, \beta)$ consists only of irreducible points, which can be counted with signs depending on a choice of orientation and homology orientation on $X$. This count, denoted $\# \mathcal{M}(X, g, \beta)$, will generally depend on the parameters $(g, \beta)$ because reducible solutions can appear along a path connecting two sets of generic parameters. To remedy this, we introduce an indextheoretic correction term $w(X, g, \beta)$ whose definition depends on an understanding of the Dirac operator on a non-compact manifold with end modeled on the infinite cyclic cover of $X$, using Taubes' theory [51] of end-periodic operators and some additional analysis. By proving that the change in $w(X, g, \beta)$ (the aforementioned spectral flow) is the same as the change in $\# \mathcal{M}(X, g, \beta)$, we arrive at the main result of this paper:

Theorem A. Let $X$ be a smooth oriented homology oriented 4-manifold with the integral homology of $S^{1} \times S^{3}$, and define

$$
\lambda_{\mathrm{SW}}(X)=\# \mathcal{M}(X, g, \beta)-w(X, g, \beta) .
$$

Then $\lambda_{\mathrm{SW}}(X)$ is independent of the choice of metric $g$ and generic perturbation $\beta$; moreover, the reduction of $\lambda_{\mathrm{SW}}(X)$ modulo 2 is the Rohlin invariant of $X$.

When $X$ has the form $S^{1} \times Y$ for an integral homology sphere $Y$, our invariant becomes the invariant of $Y$ studied by Weimin Chen [9] and Yuhan Lim [25]. Indeed, our approach was inspired by their treatment of a similar issue with the count of irreducibles in the SeibergWitten moduli space on $Y$, namely, that this count can change in a one-dimensional family of parameters. To counter this, Chen and Lim (independently, following a suggestion of Kronheimer [12]) introduced an index-theoretic, metric-dependent invariant of $Y$ that jumps in the same fashion as the above count. In fact, this 'counter-term' is a combination of $\eta$-invariants associated to the Dirac and odd signature operators on $Y$. One can say that our correction term $w(X, g, \beta)$ plays the role of this combination of $\eta$-invariants in the setting when the operators are periodic rather than $\mathbb{R}$-invariant over the end.

For an integral homology sphere $Y, \operatorname{Lim}[\mathbf{2 6}]$ proved that the invariant of $Y$ defined in this fashion actually coincides, up to an overall sign, with the Casson invariant $\lambda(Y)$. The latter is defined by counting either irreducible flat $S U(2)$ connections on $Y$ as in Taubes [50], or irreducible $S U(2)$ representations of its fundamental group; see AkbulutMcCarthy [2]. The Casson invariant was extended by Furuta and Ohta $[\mathbf{1 6}]$ to an invariant $\lambda_{\mathrm{FO}}(X)$ of smooth 4-manifolds $X$ with the 
$\mathbb{Z}[\mathbb{Z}]$-homology of $S^{1} \times S^{3}$. This invariant counts irreducible flat $S U(2)$ connections on $X$ in the sense of the Donaldson gauge theory [11]. Again, this count corresponds to a count of irreducible $S U(2)$ representations of $\pi_{1}(X)$; in particular, the invariant vanishes for the homotopy $S^{1} \times S^{3}$. The second and third authors studied $\lambda_{\mathrm{FO}}(X)$ in earlier papers $[43,45]$. Now we make the following conjecture, which we have verified in many examples.

Conjecture B. For any smooth oriented homology oriented 4-manifold $X$ with the $\mathbb{Z}[\mathbb{Z}]$-homology of $S^{1} \times S^{3}$, one has

$$
\lambda_{\mathrm{SW}}(X)=-\lambda_{\mathrm{FO}}(X) .
$$

If this conjecture turns out to be true, it will answer several longstanding questions in topology, the most striking of which is the vanishing of the Rohlin invariant for a homotopy $S^{1} \times S^{3}$. See [43] for a longer discussion.

Finally, we point out that Seiberg-Witten and Yang-Mills invariants for manifolds with $b_{+}^{2}=0$ have also been considered by other authors. Okonek-Teleman $[\mathbf{3 9}]$ and Teleman [52] studied these in connection with problems about curves on complex surfaces of type $\mathrm{VII}_{0}$. The topological setup in these papers is more general, in that $b^{2}=b_{-}^{2} \neq 0$, and $b^{1}$ can be bigger than 1 . A related preprint of Lobb-Zentner [27] treats the case of negative definite 4 -manifolds with non-zero $b^{2}$ divisible by 4 and $b^{1}=1$, and defines an invariant by counting projectively flat $S U(2)$ connections; Zentner [54] has subsequently shown that this invariant actually vanishes. Recently, Frøyshov [14] has studied two numerical invariants of a negative definite 4 -manifold $X$ with $b^{1}(X)=1$. One invariant is the Lefschetz number of a cobordism induced map on a version of the monopole Floer homology; its definition requires that $X$ has an embedded rational homology sphere $Y$ generating $H_{3}(X ; \mathbb{Z})$. The other invariant is the $h$-invariant, whose definition requires that either $b^{2}(X)=0$ or a generator of $H_{3}(X ; \mathbb{Z})$ is represented by an integral homology sphere $Y$. It would be of interest to understand the relation of all of these works to the present paper. We show that our definition of the invariant $\lambda_{\mathrm{SW}}(X)$ can be extended to negative definite manifolds $X$ with $b_{-}^{2}(X) \neq 0$, at least under the assumption that either $b_{-}^{2}(X)=1$ or else there is a homology sphere $Y$ generating $H_{3}(X ; \mathbb{Z})$; this extension is described in Section 10.

Here is a brief outline of the paper. We begin in Section 2 with a description of the blown-up Seiberg-Witten moduli space introduced in [23] to deal with reducible solutions, and establish some transversality results for 1-parameter moduli spaces that will be used later in the paper. In Section 3, we introduce the Dirac operator on periodic-end manifolds, and define the correction term $w(X, g, \beta)$. Section 4 contains the basic analysis of the Dirac operator on the infinite cyclic cover $\tilde{X}$ of a 
manifold $X$, including its Fredholm properties when acting on weighted Sobolev spaces $L_{k, \delta}^{2}(\tilde{X})$. The main tool here is the Fourier-Laplace transform defined in $[\mathbf{5 1}]$ that relates this periodic Dirac operator on $\tilde{X}$ to a family of twisted Dirac operators on $X$ with parameter $z \in \mathbb{C}^{*}$. We show that the inverses of this family depend meromorphically on $z$, and describe the variation of the poles in a generic path of metrics and perturbations. In the following Sections 5 and 6, we extend this analysis to the case of a manifold with a periodic end modeled on $\tilde{X}$, establishing asymptotics for solutions to the Dirac equation and a change of index formula. This formula is applied in Section 7 to interpret the index change as a spectral flow. Finally, in Section 8, we put together the results of the preceding sections to match the change in $w(X, g, \beta)$ with jumps in $\# \mathcal{M}(X, g, \beta)$ in a generic 1-parameter family of metrics and perturbations, and hence to prove the independence of $\lambda_{\mathrm{SW}}(X)$ from the choice of metric and generic perturbation. Section 9 contains the proof that $\lambda_{\mathrm{SW}}(X)$ reduces modulo 2 to the Rohlin invariant; this involves a careful choice of metric as in [44] and perturbation that is equivariant with respect to the well-known involution in Seiberg-Witten theory. Section 10 describes an extension of $\lambda_{\mathrm{SW}}(X)$ to certain negative definite manifolds $X$ with $b^{1}(X)=1$. The final Section 11 provides some examples of explicit calculations; we remark that all of these are in agreement with Conjecture B.

Acknowledgments. We thank Kim Frøyshov, Lev Kapitanski, Claude LeBrun, Leonid Parnovski, and Cliff Taubes for useful discussions and for sharing their expertise. We also thank the referee for some perceptive comments and suggestions.

The first author was partially supported by NSF Grant 0805841, the second author was partially supported by NSF Grant 0804760, and the third author was partially supported by NSF Grant 0305946 and the Max-Planck-Institut für Mathematik in Bonn, Germany.

\section{Seiberg-Witten moduli spaces}

Let $X$ be an oriented smooth homology $S^{1} \times S^{3}$, and choose a spin structure on $X$. Two different spin structures on $X$ are equivalent as $\operatorname{spin}^{c}$ structures; hence our construction will be independent of this choice. Fix a homology orientation on $X$ by fixing a generator $1 \in \mathbb{Z}=$ $H^{1}(X ; \mathbb{Z})$. In this section, we will introduce, following $[\mathbf{2 3}]$, the blownup Seiberg-Witten moduli space on $X$ and investigate its dependence on the (generic) metric and perturbation.

2.1. Definition. Given a metric $g$ on $X$ and a form $\omega \in \Omega_{+}^{2}(X, i \mathbb{R})$, consider the triples $(A, s, \varphi)$ composed of a $U(1)$-connection $A$ on the 
determinant bundle of the spin bundle, a real number $s \geq 0$, and a positive spinor $\varphi$ such that $\|\varphi\|_{L^{2}}=1$. We will slightly abuse notation by viewing $A$ as a form in $\Omega^{1}(X, i \mathbb{R})$ after fixing a trivialization of the determinant bundle. The gauge group $\mathcal{G}=\operatorname{Map}\left(X, S^{1}\right)$ acts freely on such triples by the rule $u(A, s, \varphi)=\left(A-u^{-1} d u, s, u \varphi\right)$. The (perturbed, blown-up) Seiberg-Witten moduli space consists of the gauge equivalence classes of the triples $(A, s, \varphi)$ that solve the Seiberg-Witten equations

$$
\left\{\begin{array}{l}
F_{A}^{+}-s^{2} \tau(\varphi)=\omega \\
D_{A}^{+}(X, g)(\varphi)=0 .
\end{array}\right.
$$

The fact that $b_{+}^{2}(X)=0$ allows for the following description of the forms $\omega \in \Omega_{+}^{2}(X, i \mathbb{R})$. We use the standard notation $\mathcal{H}^{k}$ for harmonic $k$-forms.

Lemma 2.1. For any $\omega \in \Omega_{+}^{2}(X, i \mathbb{R})$ there exists a unique $\beta \in$ $\Omega^{1}(X, i \mathbb{R})$ such that $d^{+} \beta=\omega, d^{*} \beta=0$, and $\beta$ is orthogonal to $\mathcal{H}^{1}(X ; i \mathbb{R})$ $\subset \Omega^{1}(X, i \mathbb{R})$.

Proof. Since $H_{+}^{2}(X ; i \mathbb{R})=0$, the Hodge decomposition for $\omega$ takes the form $\omega=d \alpha+d^{*} \gamma=d \alpha+* d \beta$ where $\beta=* \gamma$. Since $* \omega=\omega$, we have $\omega=d \beta+* d \alpha$. By the uniqueness of the Hodge decomposition, $\alpha=\beta$ and hence $\omega=d \beta+* d \beta=d^{+} \beta$. One can of course choose $\beta \in(\operatorname{im} d)^{\perp}=\operatorname{ker} d^{*}$, which makes it unique up to adding a harmonic 1 -form in $\mathcal{H}^{1}(X ; i \mathbb{R})$.

q.e.d.

In other words, we have a linear isomorphism $d^{+}: \mathcal{P} \rightarrow \Omega_{+}^{2}(X, i \mathbb{R})$ with $\mathcal{P}=\operatorname{ker} d^{*} \cap \mathcal{H}^{1}(X ; i \mathbb{R})^{\perp}$. The vector space $\mathcal{P}$ will be referred to as the space of perturbations. Given a perturbation $\beta \in \mathcal{P}$, the SeibergWitten moduli space corresponding to $\omega=d^{+} \beta$ in (1) will be denoted $\mathcal{M}(X, g, \beta)$.

2.2. Regularity. Fix an integer $k \geq 3$, and define the (blown-up) configuration space $\widetilde{\mathcal{B}}$ as the Hilbert manifold of the $L_{k+1}^{2}$ gauge equivalence classes of triples $(A, s, \varphi)$, where $A$ is a $U(1)$-connection on $X$ of Sobolev class $L_{k}^{2}, s$ is a real number, and $\varphi$ is a positive $L_{k}^{2}$ spinor such that $\|\varphi\|_{L^{2}}=1$. The space $\widetilde{\mathcal{B}}$ has an involution that sends $(A, s, \varphi)$ to $(A,-s, \varphi)$. We define the configuration space $\mathcal{B}$ as the subset of $\widetilde{\mathcal{B}}$ where $s \geq 0$.

Inside the configuration space $\widetilde{\mathcal{B}}$ sits the Hilbert submanifold $\widetilde{\mathcal{Z}}$ of the gauge equivalence classes of triples $(A, s, \varphi)$ with $D_{A}^{+}(X, g)(\varphi)=0$; see [23, lemma 27.1.1]. Again, $\widetilde{\mathcal{Z}}$ has the natural involution $(A, s, \varphi) \rightarrow$ $(A,-s, \varphi)$. The condition $s \geq 0$ defines $\mathcal{Z} \subset \mathcal{B}$, a Hilbert submanifold with boundary. The coordinate $s$ is a product factor, and the boundary $\partial \mathcal{Z}$ of $\mathcal{Z}$ occurs at $s=0$. 
Let the map $\chi: \widetilde{\mathcal{Z}} \rightarrow \Omega_{+}^{2}(X, i \mathbb{R})$ be given by $\chi(A, s, \varphi)=F_{A}^{+}-$ $s^{2} \tau(\varphi)$, where $\Omega_{+}^{2}(X, i \mathbb{R})$ is completed in the Sobolev $L_{k-1}^{2}$ norm. (This map is called $\varpi$ in $[\mathbf{2 3}]$.) For any $\beta$ in the space $\mathcal{P}$ completed in the Sobolev $L_{k}^{2}$ norm, define

$$
\widetilde{\mathcal{M}}(X, g, \beta)=\chi^{-1}\left(d^{+} \beta\right) \subset \widetilde{\mathcal{Z}} .
$$

The map $\chi$ is invariant with respect to the involution $(A, s, \varphi) \rightarrow$ $(A,-s, \varphi)$; hence we have an induced involution on $\widetilde{\mathcal{M}}(X, g, \beta)$, and the Seiberg-Witten moduli space $\mathcal{M}(X, g, \beta)$ defined in the previous section is the intersection $\widetilde{\mathcal{M}}(X, g, \beta) \cap \mathcal{Z}$. Also define

$$
\mathcal{M}^{0}(X, g, \beta)=\widetilde{\mathcal{M}}(X, g, \beta) \cap \partial \mathcal{Z} ;
$$

the points in $\mathcal{M}^{0}(X, g, \beta)$ will be called reducible.

Proposition 2.2. For a generic $\beta \in \mathcal{P}$, the moduli space $\mathcal{M}(X, g, \beta)$ $\subset \mathcal{Z}$ is a compact oriented manifold of dimension zero with empty $\mathcal{M}^{0}(X, g, \beta)$.

Proof. This is essentially contained in [23, lemma 27.1.1]. The map $\chi: \widetilde{\mathcal{Z}} \rightarrow \Omega_{+}^{2}(X, i \mathbb{R})$ is Fredholm of index zero, and its restriction $\partial \chi: \partial \mathcal{Z} \rightarrow \Omega_{+}^{2}(X, i \mathbb{R})$ is Fredholm of index -1 . Therefore, for generic $\beta$, $\widetilde{\mathcal{M}}(X, g, \beta)=\chi^{-1}\left(d^{+} \beta\right)$ is a manifold of dimension zero and $\mathcal{M}^{0}(X, g, \beta)$ $=(\partial \chi)^{-1}\left(d^{+} \beta\right)$ is empty. The moduli space $\mathcal{M}(X, g, \beta)$ is compact, and it is oriented as usual by the choice of orientation and homology orientation on $X$.

q.e.d.

Any pair $(g, \beta)$ provided by Proposition 2.2 will be called regular. Given a regular pair $(g, \beta)$, denote by $\# \mathcal{M}(X, g, \beta)$ the signed count of points in the (regular) moduli space $\mathcal{M}(X, g, \beta)$.

2.3. Regularity in families. Let $g_{I}$ be a smooth path of metrics on $X$ parameterized by $I=[0,1]$, and consider the parameterized configuration space

$$
\widetilde{\mathcal{B}}_{I}=\bigcup_{t \in I}\{t\} \times \widetilde{\mathcal{B}}_{t}
$$

where $\widetilde{\mathcal{B}}_{t}$ stands for a copy of $\widetilde{\mathcal{B}}$ with respect to the metric $g_{t}$. We will follow [23, page 479$]$ and slightly abuse notations in regarding $\widetilde{\mathcal{B}}_{t}$ as independent of $t$ and identifying $\widetilde{\mathcal{B}}_{I}$ with $I \times \widetilde{\mathcal{B}}_{0}$.

Remark 2.3. The precise identification is as follows; cf. [44, section 3.1]. For any $t \in I$, there is a unique automorphism $b_{t}: T X \rightarrow T X$ that is positive, symmetric with respect to $g_{0}$, and has the property that $g_{0}(u, v)=g_{t}\left(b_{t}(u), b_{t}(v)\right)$. The map on orthonormal frames induced by $b_{t}$ gives rise to a map $\bar{b}_{t}: S_{0}^{ \pm} \rightarrow S_{t}^{ \pm}$of spinor bundles associated with metrics $g_{0}$ and $g_{t}$. This map is an isomorphism preserving the fiberwise length of spinors. The identification $I \times \widetilde{\mathcal{B}}_{0} \rightarrow \widetilde{\mathcal{B}}_{I}$ is then given by $(t, A, s, \varphi) \rightarrow\left(t, A, s, \bar{b}_{t}(\varphi)\right)$. 


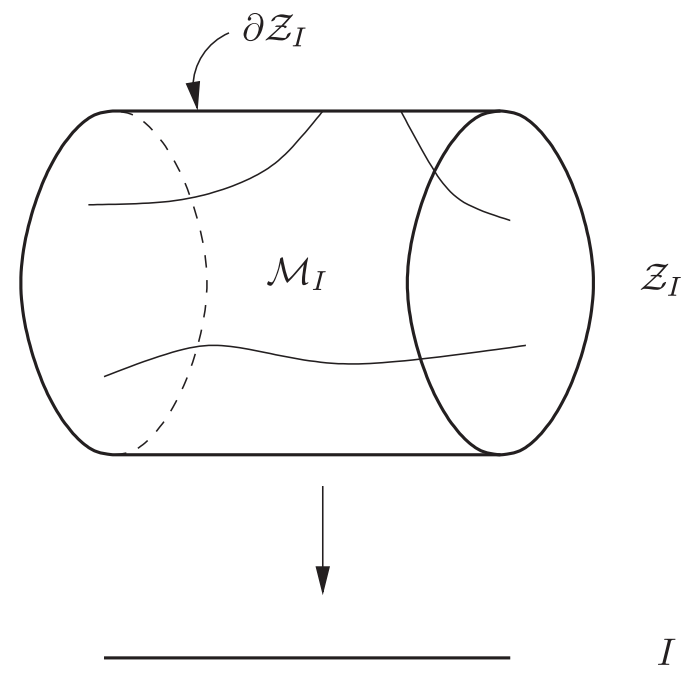

Figure 1. Parameterized moduli space

Let $\widetilde{\mathcal{Z}}_{t} \subset \widetilde{\mathcal{B}}_{t}$ be given by $D_{A}^{+}\left(X, g_{t}\right)(\varphi)=0$. The spaces $\widetilde{\mathcal{Z}}_{t}$ may be assembled into a Hilbert manifold

$$
\widetilde{\mathcal{Z}}_{I}=\bigcup_{t \in I}\{t\} \times \widetilde{\mathcal{Z}}_{t} \subset \widetilde{\mathcal{B}}_{I}
$$

over $I$ with a natural projection $\pi: \widetilde{\mathcal{Z}}_{I} \rightarrow I$. Both $\widetilde{\mathcal{B}}_{I}$ and $\widetilde{\mathcal{Z}}_{I}$ admit an involution sending $(t, A, s, \varphi)$ to $(t, A,-s, \varphi)$. The conditions $s \geq 0$ and $s=0$ define, respectively, subbundles $\mathcal{Z}_{I} \subset \widetilde{\mathcal{Z}}_{I}$ and $\partial \mathcal{Z}_{I} \subset \mathcal{Z}_{I}$ over $I$; see Figure 1.

Let $\Omega_{I}$ be the subspace of $I \times \Omega^{2}(X, i \mathbb{R})$ comprised of the pairs $(t, \omega)$ such that $\omega$ is self-dual with respect to the metric $g_{t}$. Then we have a fibration $\pi: \Omega_{I} \rightarrow I$ that can be trivialized using the maps $b_{t}$ as in Remark 2.3. Consider the commutative diagram

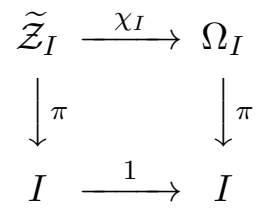

where the map $\chi_{I}$ is given by $\chi_{I}(t, A, s, \varphi)=\left(t, F_{A}^{+t}-s^{2} \tau_{t}(\varphi)\right)$. The pre-image of a section $\omega_{I}=d^{+} \beta_{I}: I \rightarrow \Omega_{I}$ under $\chi_{I}$ is the parameterized moduli space

$$
\widetilde{\mathcal{M}}_{I}=\bigcup_{t \in I}\{t\} \times \widetilde{\mathcal{M}}\left(X, g_{t}, \beta_{t}\right)
$$


Let us also consider

$$
\mathcal{M}_{I}=\widetilde{\mathcal{M}}_{I} \cap \mathcal{Z}_{I}=\bigcup_{t \in I}\{t\} \times \mathcal{M}\left(X, g_{t}, \beta_{t}\right)
$$

and

$$
\mathcal{M}_{I}^{0}=\widetilde{\mathcal{M}}_{I} \cap \partial \mathcal{Z}_{I}=\bigcup_{t \in I}\{t\} \times \mathcal{M}^{0}\left(X, g_{t}, \beta_{t}\right) .
$$

Theorem 2.4. Let $g_{I}$ be a path of metrics and $\beta_{0}, \beta_{1} \in \mathcal{P}$ a pair of perturbations such that $\mathcal{M}\left(X, g_{0}, \beta_{0}\right)$ and $\mathcal{M}\left(X, g_{1}, \beta_{1}\right)$ are regular. Then, for a generic path $\beta_{t} \in \mathcal{P}$ connecting $\beta_{0}$ to $\beta_{1}$, the parameterized moduli space $\mathcal{M}_{I}$ is a smooth oriented 1-manifold with oriented boundary

In particular,

$$
\mathcal{M}\left(X, g_{0}, \beta_{0}\right) \cup \mathcal{M}\left(X, g_{1}, \beta_{1}\right) \cup \mathcal{M}_{I}^{0} .
$$

$$
\# \mathcal{M}\left(X, g_{1}, \beta_{1}\right)-\# \mathcal{M}\left(X, g_{0}, \beta_{0}\right)=\# \mathcal{M}_{I}^{0},
$$

where $\# \mathcal{M}_{I}^{0}$ stands for the signed count of points in $\mathcal{M}_{I}^{0}$.

Proof. Observe that $\chi_{I}$ is a Fredholm map of index zero, and choose an arbitrary section $\omega_{I}: I \rightarrow \Omega_{I}$ that restricts to $\omega_{0}=d^{+} \beta_{0}$ and $\omega_{1}=$ $d^{+} \beta_{1}$ at the endpoints of $I$. According to [49, theorem 3.1], it can be approximated by a section, called again $\omega_{I}=d^{+} \beta_{I}$, that is transversal to both $\partial \chi_{I}: \partial \mathcal{Z}_{I} \rightarrow \Omega_{I}$ and $\chi_{I}: \widetilde{\mathcal{Z}}_{I} \rightarrow \Omega_{I}$. Then $\widetilde{\mathcal{M}}_{I}=\chi_{I}^{-1}\left(d^{+} \beta_{I}\right)$ is a regular oriented 1-manifold with boundary $\widetilde{\mathcal{M}}\left(X, g_{0}, \beta_{0}\right) \cup \widetilde{\mathcal{M}}\left(X, g_{1}, \beta_{1}\right)$, and $\mathcal{M}_{I}$ is a regular oriented 1-manifold whose boundary is as claimed.

q.e.d.

Any path $\left(g_{I}, \beta_{I}\right)$ with regular endpoints provided by Theorem 2.4 will be called regular. We will wish to calculate $\# \mathcal{M}_{I}^{0}$ and, eventually, compare it with the jumps in the index of a certain differential operator; see Theorem 8.1. The calculation of the index change will be greatly simplified if the regular path $\left(g_{I}, \beta_{I}\right)$ is chosen so that $g_{I}$ is constant near each $t \in I$ where $\mathcal{M}^{0}\left(X, g_{t}, \beta_{t}\right)$ is non-empty; such a path will be called special. In the Appendix, we will prove the following result.

Theorem 2.5. Any regular path $\left(g_{I}, \beta_{I}\right)$ can be homotoped rel its endpoints to a special path.

2.4. The reducibles. It will be useful to have a more explicit description of $\mathcal{M}_{I}^{0}$, as much of this paper is concerned with the behavior of the Seiberg-Witten equations at reducible points. For any choice of $(g, \beta)$, the space $\mathcal{M}^{0}(X, g, \beta)$ consists of the gauge equivalence classes of pairs $(A, \varphi)$ such that $F_{A}^{+}=d^{+} \beta, D_{A}^{+}(X, g)(\varphi)=0$, and $\|\varphi\|_{L^{2}}=1$. The gauge equivalence classes $[A]$ of connections such that $F_{A}^{+}=d^{+} \beta$ form the circle

$$
\beta+\mathcal{H}^{1}(X ; i \mathbb{R}) / H^{1}(X ; i \mathbb{Z})
$$


For each $[A]$ on this circle such that ker $D_{A}^{+}(X, g) \neq 0$, solutions of the equation $D_{A}^{+}(X, g)(\varphi)=0$ with $\|\varphi\|_{L^{2}}=1$ form a sphere of dimension $2 \operatorname{dim}_{\mathbb{C}}$ ker $D_{A}^{+}(X, g)-1$ and, after factoring out the residual $S^{1}$ gauge symmetry, the complex projective space of (complex) dimension $\operatorname{dim}_{\mathbb{C}} \operatorname{ker} D_{A}^{+}(X, g)-1$.

In particular, for any regular $(g, \beta)$, the space $\mathcal{M}^{0}(X, g, \beta)$ is empty, which means that $\operatorname{ker} D_{A}^{+}(X, g)=0$ for all points $[A]$ on the circle (3). Furthermore, for any pair $(g, \beta)$ in a regular path $\left(g_{I}, \beta_{I}\right)$, the space $\mathcal{M}^{0}(X, g, \beta)$ is at most finite, which implies that $\operatorname{ker} D_{A}^{+}(X, g) \neq 0$ for at most finitely many points $[A]$ on the circle $(3)$, and in fact that $\operatorname{ker} D_{A}^{+}(X, g)=\mathbb{C}$ at each of them.

Parameterize the circle (3) by choosing a smooth function $f: X \rightarrow S^{1}$ such that

$$
\left[f^{*}(d \theta)\right]=1 \in H^{1}(X ; \mathbb{Z})
$$

corresponding to our choice of homology orientation on $X$. Then the above discussion can be restated as follows in terms of the family

$$
D_{A}^{+}(X, g)=D^{+}(X, g)+\beta-\ln z \cdot f^{*}(d \theta) \quad \text { with } \quad|z|=1 .
$$

Proposition 2.6. For any regular $(g, \beta)$, all operators in the family (5) have zero kernels. For any point $(g, \beta)$ in a regular path $\left(g_{I}, \beta_{I}\right)$, at most finitely many of the operators (5) have non-zero kernel, and that kernel is isomorphic to $\mathbb{C}$.

\section{The correction term}

Let $X$ be an oriented smooth spin homology $S^{1} \times S^{3}$ with a fixed homology orientation. In this section, for any regular pair $(g, \beta)$, we will introduce the correction term $w(X, g, \beta)$ and will prove that it is well defined.

3.1. End-periodic manifolds. Let $Y \subset X$ be a connected spin 3manifold dual to the generator of $H^{1}(X ; \mathbb{Z})$ so that $X$ cut open along $Y$ is a spin homology cobordism $W$ from $Y$ to itself. Corresponding to the isomorphism $H_{1}(X ; \mathbb{Z}) \rightarrow \mathbb{Z}$ is the infinite cyclic cover

$$
\tilde{X}=\ldots \cup W_{-1} \cup W_{0} \cup W_{1} \cup \ldots \text { with all } W_{n}=W .
$$

By an end-periodic (spin) manifold whose end is modeled on $\tilde{X}$ we will mean any manifold $Z_{+}=Z \cup \tilde{X}_{+}$, where $Z$ is a smooth compact spin manifold with spin boundary $\partial Z=Y$, and

$$
\tilde{X}_{+}=W_{0} \cup W_{1} \cup W_{2} \cup \ldots \text { with all } W_{n}=W .
$$

Any metric $g$ on $X$ naturally lifts to a periodic metric on $\tilde{X}_{+}$and extends to an end-periodic metric on $Z_{+}$, again called $g$. The same holds for the perturbation 1-forms $\beta \in \mathcal{P}$. 
3.2. End-periodic Dirac operators. Given an end-periodic manifold $Z_{+}$, with any choice of metric $g$, perturbation 1 -form $\beta$, and spin structure as above, one associates the perturbed Dirac operator

$$
D^{+}\left(Z_{+}, g, \beta\right):=D^{+}\left(Z_{+}, g\right)+\beta: L_{1}^{2}\left(Z_{+}, S^{+}\right) \rightarrow L^{2}\left(Z_{+}, S^{-}\right) \text {. }
$$

Theorem 3.1. The operator (7) is Fredholm for any regular pair $(g, \beta)$ on the manifold $X$.

Proof. The operator $D^{+}(X, g)+\beta$ has zero index. Therefore, according to Taubes [51, lemma 4.3], the operator (7) is Fredholm if and only if the kernels of the operators in the family (5) vanish for any choice of smooth function $f: X \rightarrow S^{1}$ satisfying (4). But for a regular $(g, \beta)$, these kernels vanish by Proposition 2.6. Note that the statement of lemma 4.3 in $[\mathbf{5 1}]$ should read $\delta \in \mathbb{R}$ rather than $\delta \in \mathbb{R}^{*}$ (interpreted to mean $\mathbb{R} \backslash\{0\}$ ), which can be confirmed by a reading of the proof of that lemma. This typo was pointed out to us by the referee.

In Sections 4 and 5 below, we will explain in detail the basic analytical machinery behind Theorem 3.1 and re-prove it in more general situation; see Corollary 5.2.

3.3. Definition of the correction term. Let $Z_{+}=Z \cup \tilde{X}_{+}$be an end-periodic manifold and $(g, \beta)$ a regular pair on $X$ so that the operator (7) is Fredholm by Theorem 3.1. Define the correction term

$$
w(X, g, \beta)=\operatorname{ind}_{\mathbb{C}} D^{+}\left(Z_{+}, g, \beta\right)+\operatorname{sign}(Z) / 8 .
$$

Note that in general $w(X, g, \beta)$ will be a rational number.

Proposition 3.2. The correction term $w(X, g, \beta)$ is independent of the choices of $Z$ and $Y \subset X$, and the way $g, \beta$, and the spin structure are extended to $Z$.

Proof. Given two choices, $Z$ and $Z^{\prime}$ with $\partial Z=\partial Z^{\prime}=Y$, we use the excision principle - see Section 5.3 - and the index theorem to obtain

$$
\begin{aligned}
& \text { ind }_{\mathbb{C}} D^{+}\left(Z_{+}^{\prime}, g, \beta\right)-\operatorname{ind}_{\mathbb{C}} D^{+}\left(Z_{+}, g, \beta\right)=\operatorname{ind}_{\mathbb{C}} D^{+}\left(-Z \cup Z^{\prime}\right) \\
& =-\frac{1}{24} \int_{-Z \cup Z^{\prime}} p_{1}=-\frac{1}{8} \operatorname{sign}\left(-Z \cup Z^{\prime}\right)=\frac{1}{8} \operatorname{sign}(Z)-\frac{1}{8} \operatorname{sign}\left(Z^{\prime}\right),
\end{aligned}
$$

which proves that $w(X, g, \beta)$ is independent of the choices of $Z$ and the extensions.

Next, let $Y$ and $Y^{\prime}$ be two choices for cutting $X$ open, and choose a lift of each to the infinite cyclic cover $\tilde{X}$. Call the lifts $Y$ and $Y^{\prime}$ again. Since $Y$ and $Y^{\prime}$ are both compact, one can apply the covering translation to $Y^{\prime}$ sufficiently many times to ensure that $Y$ and $Y^{\prime}$ are disjoint. Both $Y$ and $Y^{\prime}$ separate $\tilde{X}$; hence they become the boundary components of a spin cobordism $V \subset \tilde{X}$. We claim that the signature 
of $V$ is zero. This fact is well-known, but we do not know a precise reference; compare $[\mathbf{4 2}]$ and $[\mathbf{3 8}]$.

To verify the claim, note that $V$ is disjoint from its image under a sufficiently high prime power $p$ of the covering translation, and hence the projection of $V$ into the $p$-fold cyclic cover of $X$ is an embedding. But a standard argument in knot theory $[\mathbf{1 8}, \S 5]$ says that this $p$-fold cover is a rational homology $S^{1} \times S^{3}$, so that the intersection form on its 2-dimensional rational homology vanishes identically. It follows that the intersection form on $V$ vanishes as well, so the signature of $V$ is zero. That $w(X, g, \beta)$ is independent of the choice of $Y$ now follows by the argument in the previous paragraph applied to $Z^{\prime}=Z \cup V$. q.e.d.

\section{Dirac operators on infinite cyclic covers}

In the definition of the correction term $w(X, g, \beta)$ in Section 3, we were able to avoid most of the analysis on end-periodic manifolds by simply quoting Theorem 3.1, which is essentially due to Taubes [51]. Getting a firm grip on this analysis and, in particular, on the ideas involved in the proof of Theorem 3.1, becomes essential once we wish to prove that the difference $\# \mathcal{M}(X, g, \beta)-w(X, g, \beta)$ is independent of the choice of regular $(g, \beta)$. We will study this analysis in the next four sections.

This section is dedicated to the Fourier-Laplace transform (following Taubes [51]) and the role it plays in establishing Fredholmness of the Dirac operator on the infinite cyclic cover $\tilde{X} \rightarrow X$. The same transform arises in related contexts; for example, the terminology ' $Z$-transform' is widely used in the literature (e.g. [20]). A number of proofs in this section are standard, and are largely omitted.

4.1. Infinite cyclic cover. Let $X$ be an oriented smooth spin homology $S^{1} \times S^{3}$ with a fixed homology orientation, and $f: X \rightarrow S^{1}$ a smooth function satisfying (4). Let $\tilde{X}$ be the infinite cyclic cover as in (6) and $\tilde{f}: \tilde{X} \rightarrow \mathbb{R}$ a lift of $f$ such that $\tilde{f}(x+n)=\tilde{f}(x)+n$. Here, $x \mapsto x+1$ stands for the covering translation $\tilde{X} \rightarrow \tilde{X}$. Any metric $g$ on $X$ lifts to a periodic metric on $\tilde{X}$ again called $g$, and the same holds for the spin structure.

Note that in the product case, when $X=S^{1} \times Y$ with the product metric and spin structure, and $Y$ is an integral homology 3-sphere, one has $\tilde{X}=\mathbb{R} \times Y$. The function $\tilde{f}: \mathbb{R} \times Y \rightarrow \mathbb{R}$ can then be chosen to be $\tilde{f}(\theta, y)=\theta$.

4.2. Definition of the Fourier-Laplace transform. Given a spinor $u \in C_{0}^{\infty}\left(\tilde{X}, S^{ \pm}\right)$and a complex number $\mu \in \mathbb{C}$, the Fourier-Laplace transform of $u$ is defined as

$$
\hat{u}_{\mu}(x)=e^{\mu \tilde{f}(x)} \sum_{n=-\infty}^{\infty} e^{\mu n} u(x+n),
$$


where $x+n$ denotes the result of applying to $x \in \tilde{X}$ the covering translation $n$ times. Since $u$ has compact support, the above sum is finite. One can easily check that $\hat{u}_{\mu}(x+1)=\hat{u}_{\mu}(x)$ for all $x \in \tilde{X}$. Therefore, for every $\mu \in \mathbb{C}$, we have a well-defined spinor $\hat{u}_{\mu}$ over $X$. Note that the spinor $\hat{u}_{\mu}$ depends analytically on $\mu$, and that for any $x \in X$, we have

$$
\hat{u}_{\mu+2 \pi i}(x)=e^{2 \pi i f(x)} \hat{u}_{\mu}(x) .
$$

In order to recover $u$ from its Fourier-Laplace transform, we do not need to know $\hat{u}_{\mu}$ for all $\mu \in \mathbb{C}$; in fact, it suffices to know $\hat{u}_{\mu}$ for $\mu$ in just one interval of the form $I(\nu)=\{\nu+i \theta \mid 0 \leq \theta \leq 2 \pi\}$ with $\nu \in \mathbb{C}$. The formula is as follows:

$$
u(x+n)=\frac{1}{2 \pi i} \int_{I(\nu)} e^{-\mu(f(x)+n)} \hat{u}_{\mu}(x) d \mu,
$$

where $x \in W_{0}$ and $n$ is an arbitrary integer. This can be checked by direct substitution.

4.3. The Fourier-Laplace transform in weighted Sobolev spaces. Given $\delta \in \mathbb{R}$ and an integer $k \geq 0$, we will say that $u \in L_{k, \delta}^{2}\left(\tilde{X}, S^{ \pm}\right)$if and only if $e^{\delta \tilde{f}} u \in L_{k}^{2}\left(\tilde{X}, S^{ \pm}\right)$, and let

$$
\|u\|_{L_{k, \delta}^{2}\left(\tilde{X}, S^{ \pm}\right)}=\left\|e^{\delta \tilde{f}} u\right\|_{L_{k}^{2}\left(\tilde{X}, S^{ \pm}\right)} .
$$

We wish to extend our definition of the Fourier-Laplace transform to these weighted Sobolev spaces. For the sake of brevity, we will often omit $S^{ \pm}$from our notations and write $L^{2}(\tilde{X})$ for $L^{2}\left(\tilde{X}, S^{ \pm}\right)$, etc. The basic observations are summarized as follows.

Proposition 4.1. For any $u \in C_{0}^{\infty}(\tilde{X})$ and $\nu \in \mathbb{C}$, the restriction of the family $\hat{u}_{\mu}$ to the interval $I(\nu)$ belongs to the Hilbert space $L^{2}\left(I(\nu), L_{k}^{2}(X)\right)$. Moreover, there is a constant $C$ such that

$$
\left\|\left.\hat{u}_{\mu}\right|_{I(\nu)}\right\|_{L^{2}\left(I(\nu), L_{k}^{2}(X)\right)}^{2} \leq C \cdot\|u\|_{L_{k, \delta}^{2}(\tilde{X})}^{2} \quad \text { with } \quad \delta=\operatorname{Re}(\nu) .
$$

Proposition 4.1 is proved using standard arguments of Fourier analysis (see for instance [40, page 290]) that readily extend to the endperiodic case. It shows that, for any $\nu \in \mathbb{C}$ with $\operatorname{Re}(\nu)=\delta \in \mathbb{R}$, the assignment $\left.u \mapsto \hat{u}_{\mu}\right|_{I(\nu)}$ can be uniquely extended to bounded linear operators

$$
A(\nu): L_{k, \delta}^{2}(\tilde{X}) \rightarrow L^{2}\left(I(\nu), L_{k}^{2}(X)\right), \quad k \in \mathbb{Z}_{+} .
$$

The following proposition is proved using formula (9) and the Parseval relation; see [40, page 290] and [34, lemma 2] in the product case.

Proposition 4.2. For any $\nu \in \mathbb{C}$ the operators (10) are linear isomorphisms. 
Finally, for use in Section 6.2, it will be helpful to know that the Fourier-Laplace transform of a spinor in a weighted Sobolev space is holomorphic in a specific region $V \subset \mathbb{C}$. We use the term holomorphic to mean that the function $V \rightarrow L_{k}^{2}(X)$ that assigns $\hat{u}_{\mu}$ to $\mu$ can be expressed as a power series in $\mu$ convergent in the $L_{k}^{2}(X)$ norm. There are many possible statements along these lines; we will content ourselves with the following result.

Lemma 4.3. Suppose that $u \in L_{\delta}^{2}(\tilde{X})$ is a smooth spinor. If $u$ has support in $\tilde{X}_{+}=W_{0} \cup W_{1} \cup \ldots$ then $\hat{u}_{\mu}$ is holomorphic in the half plane $\operatorname{Re} \mu<\delta$.

Proof. It suffices to consider the case of a smooth spinor $u \in L^{2}(\tilde{X})$ with support in $\tilde{X}_{+}$. To show that $\hat{u}_{\mu}$ is holomorphic in the half plane $\operatorname{Re} \mu<0$, all we need to do is estimate, for $x \in W_{0}$, the $L^{2}$-norm of the tail

$$
\sum_{n \geq N} e^{\mu n} u(x+n)
$$

The latter norm can be estimated by

$$
\left(\sum\left|e^{2 \mu n}\right|\right)^{1 / 2} \cdot\left(\sum\|u(x+n)\|_{L^{2}}^{2}\right)^{1 / 2}
$$

using the Hölder inequality. The first series here converges as long as $\operatorname{Re} \mu<0$ and approaches 0 as $N \rightarrow \infty$, and the second is estimated from above by $\|u\|_{L^{2}(\tilde{X})}$.

q.e.d.

4.4. The Fourier-Laplace transform of perturbed Dirac operators. Let $\beta \in \mathcal{P}$ be a perturbation 1-form pulled back to $\tilde{X}$. We will be interested in the Fredholm properties of the perturbed Dirac operator

$$
D^{+}(\tilde{X}, g, \beta):=D^{+}(\tilde{X}, g)+\beta: L_{1, \delta}^{2}\left(\tilde{X}, S^{+}\right) \rightarrow L_{\delta}^{2}\left(\tilde{X}, S^{-}\right)
$$

in weighted Sobolev spaces. The Fourier-Laplace transform of such an operator is the family of operators, parameterized by $\mu \in \mathbb{C}$, obtained by conjugating the operator by the Fourier-Laplace transform. A straightforward calculation shows that the Fourier-Laplace transform of $D^{+}(\tilde{X}, g, \beta)$ is the holomorphic family

$$
D_{\mu}^{+}(X, g, \beta)=D^{+}(X, g, \beta)-\mu \cdot f^{*}(d \theta)
$$

of perturbed Dirac operators on $X$ with $\mu \in \mathbb{C}$ (compare with (5)). Here, we use notation

$$
D^{+}(X, g, \beta):=D^{+}(X, g)+\beta
$$

for the perturbed Dirac operator on $X$. Similar formulas hold for the full Dirac operator and for the negative chiral Dirac operator.

Proposition 4.4. The operator (11) is Fredholm if and only if the operators $D_{\mu}^{+}(X, g, \beta)$ are invertible for all $\mu$ with $\operatorname{Re} \mu=\delta$. 
Proof. Since $D^{+}(X, g, \beta)$ is an elliptic operator of index zero, the statement follows from Taubes [51, lemma 4.3].

q.e.d.

Therefore, to understand the index theory of the perturbed Dirac operator (11), we need to study the family (12). The subset $\Sigma(g, \beta)$ of the complex plane consisting of all $\mu \in \mathbb{C}$ for which $D_{\mu}^{+}(X, g, \beta)$ is not invertible will be called the spectral set of the family $D_{\mu}^{+}(X, g, \beta)$.

Lemma 4.5. The spectral set $\Sigma(g, \beta)$ is independent of the choice of the function $f: X \rightarrow S^{1}$.

Proof. For any two choices of $f$, the 1 -forms $f^{*}(d \theta)$ differ by an exact form $d h$, where $h: X \rightarrow \mathbb{R}$ is a smooth function. The result now follows from the easily verified formula $D_{\mu}^{+}(X, g, \beta)-\mu d h=$ $e^{\mu h} D_{\mu}^{+}(X, g, \beta) e^{-\mu h}$.

q.e.d.

Theorem 4.6. Let $\left(g_{I}, \beta_{I}\right)$ be a regular path. For any $t \in I$, the spectral set $\Sigma\left(g_{t}, \beta_{t}\right)$ is a discrete subset of the complex plane, and the inverse of $D_{\mu}^{+}\left(X, g_{t}, \beta_{t}\right)$ is a meromorphic function of $\mu \in \mathbb{C}$.

Proof. According to Proposition 2.6, the spectral set $\Sigma\left(g_{t}, \beta_{t}\right)$ is a proper subset of $\mathbb{C}$ for any $t \in I$. Having noted this, abstract out the salient features of our situation and view $D_{\mu}^{+}\left(X, g_{t}, \beta_{t}\right)$ for any fixed $t \in I$ as a family of the shape

$$
T+\mu A: L_{1}^{2}\left(X, S^{+}\right) \rightarrow L^{2}\left(X, S^{-}\right),
$$

where $T$ (the operator $D^{+}\left(X, g_{t}, \beta_{t}\right)$ in our case) is an index zero Fredholm operator, and $A$ (Clifford multiplication by $-f_{t}^{*}(d \theta)$ ) is a compact operator. Fix $\mu_{0}$ such that the operator $T+\mu_{0} A$ is invertible.

Consider the operator $(T+\mu A)\left(T+\mu_{0} A\right)^{-1}: L^{2}\left(X, S^{-}\right) \rightarrow L^{2}\left(X, S^{-}\right)$. This is a bounded operator, and we can rewrite it as

$$
I+\left(\mu-\mu_{0}\right) A\left(T+\mu_{0} A\right)^{-1}=I+\left(\mu-\mu_{0}\right) K .
$$

The operator $K=A\left(T+\mu_{0} A\right)^{-1}: L^{2}\left(X, S^{-}\right) \rightarrow L^{2}\left(X, S^{-}\right)$is compact since both $A: L^{2} \rightarrow L^{2}$ and $\left(T+\mu_{0} A\right)^{-1}: L^{2} \rightarrow L_{1}^{2}$ are bounded so that their composition factors through the compact embedding $L_{1}^{2} \rightarrow L^{2}$. Thus we can apply the spectral theory of compact operators to the study of our family. For $\mu \neq \mu_{0}$ we conclude that $T+\mu A$ is invertible if and only if $\zeta=-\left(\mu-\mu_{0}\right)^{-1}$ is not in the spectrum of $K$. The spectrum of $K$ is a compact subset $\operatorname{Spec}(K)$ of the complex plane with only 0 as an accumulation point. Thus the spectral set is discrete.

Furthermore, the resolvent $(K-\zeta I)^{-1}$ of a compact operator is meromorphic in $\zeta=-1 /\left(\mu-\mu_{0}\right)$ away from $\zeta=0$, hence the inverse of $T+\mu A$ is meromorphic in $\mu \in \mathbb{C}$.

q.e.d.

Corollary 4.7. Let $\left(g_{I}, \beta_{I}\right)$ be a regular path. For any $t \in I$, the operator $D^{+}\left(\tilde{X}, g_{t}, \beta_{t}\right): L_{1, \delta}^{2}\left(\tilde{X}, S^{+}\right) \rightarrow L_{\delta}^{2}\left(\tilde{X}, S^{-}\right)$is Fredholm for all but a discrete set of $\delta \in \mathbb{R}$ with no accumulation points. 
The set of $\delta$ 's for which the above operator fails to be Fredholm may well depend on $t$; this dependence is examined in more detail in the next section.

4.5. Spectral set as a function of $t$. Given a path $\left(g_{I}, \beta_{I}\right)$ of metrics and perturbations on $X$, consider the parameterized spectral set

$$
\Sigma_{I}=\bigcup_{t \in I} \Sigma\left(g_{t}, \beta_{t}\right) \subset \mathbb{C} .
$$

Let $t \in I$ be such that the spectral set $\Sigma\left(g_{t}, \beta_{t}\right)$ is non-empty and, for any $\mu_{j} \in \Sigma\left(g_{t}, \beta_{t}\right)$, consider the operator

$$
P_{\mu_{j}}=\frac{1}{2 \pi i} \oint_{\Gamma}\left(D^{+}\left(X, g_{t}, \beta_{t}\right)-\mu \cdot f_{t}^{*}(d \theta)\right)^{-1} d \mu,
$$

where $\Gamma$ is a small loop in the $\mu$-plane encircling $\mu_{j}$ once in the positive direction.

Theorem 4.8. Let $\left(g_{I}, \beta_{I}\right)$ be a regular path and suppose that $\mu_{j} \in$ $\Sigma\left(g_{t}, \beta_{t}\right)$ is such that the rank of the operator (14) is one. Then there exist an open neighborhood $U\left(\mu_{j}\right)$ and a real $\varepsilon>0$ such that the intersection

$$
\bigcup_{|t-s|<\varepsilon} \Sigma\left(g_{s}, \beta_{s}\right) \cap U\left(\mu_{j}\right)
$$

is an embedded curve.

Proof. We will use notations from the proof of Theorem 4.6 and recall from that proof that the spectral set of $T+\mu A$ coincides, up to shift and inversion, with the spectrum of the compact operator $K$. The operator (14) then takes the form

$$
P_{\mu_{j}}=\frac{1}{2 \pi i} \oint_{\Gamma}(T+\mu A)^{-1} d \mu .
$$

Write $(T+\mu A)^{-1}=-\left(T+\mu_{0} A\right)^{-1} \zeta(K-\zeta I)^{-1}$ with $\zeta=-\left(\mu-\mu_{0}\right)^{-1}$; then, after changing coordinates,

$$
P_{\mu_{j}}=\left(T+\mu_{0} A\right)^{-1} \frac{1}{2 \pi i} \oint_{\Gamma^{\prime}} \zeta^{-1}(K-\zeta I)^{-1} d \zeta=\left(T+\mu_{0} A\right)^{-1} \zeta_{j}^{-1} \Pi_{\zeta_{j}}
$$

where $\Gamma^{\prime}$ is a small loop in the $\zeta$-plane encircling $\zeta_{j}=-\left(\mu_{j}-\mu_{0}\right)^{-1}$ once in the positive direction, and $\Pi_{\zeta_{j}}$ is the projector onto the generalized eigenspace of $K$ corresponding to $\zeta_{j}$. Then $\operatorname{rank}\left(\Pi_{\zeta_{j}}\right)=\operatorname{rank}\left(P_{\mu_{j}}\right)=1$, and the result follows from the perturbation theory of compact operators [21, theorem VII.1.8]

q.e.d.

Remark 4.9. We will show later in Proposition 7.2 that, for any special path $\left(g_{I}, \beta_{I}\right)$, the condition of Theorem 4.8 on the rank of the operator (14) is automatically satisfied for all $\mu_{j} \in i \mathbb{R} \cap \Sigma_{I}$. 


\section{Dirac operators on end-periodic manifolds}

In this section, we extend the results obtained in Section 4 for Dirac operators on infinite cyclic covers to Dirac operators on general manifolds with periodic ends. Taubes' paper [51] is again the basic reference; a rather different geometric application of end-periodic operators may be found in $[\mathbf{3 0}]$.

5.1. Weighted Sobolev spaces. Let $Z_{+}$be an end-periodic manifold as defined in Section 3.1, and $f: X \rightarrow S^{1}$ a smooth function satisfying (4) lifted to a function $\tilde{f}: \tilde{X} \rightarrow \mathbb{R}$ as in Section 4.1. Given $\delta \in \mathbb{R}$, extend the function $\delta \cdot \tilde{f}(x): \tilde{X}_{+} \rightarrow \mathbb{R}$ to a smooth function $h: Z_{+} \rightarrow \mathbb{R}$. We will say that $\varphi \in L_{k, \delta}^{2}\left(Z_{+}, S^{ \pm}\right)$if and only if $e^{h} \varphi \in L_{k}^{2}\left(Z_{+}, S^{ \pm}\right)$, with

$$
\|\varphi\|_{L_{k, \delta}^{2}\left(Z_{+}, S^{ \pm}\right)}=\left\|e^{h} \varphi\right\|_{L_{k}^{2}\left(Z_{+}, S^{ \pm}\right)} .
$$

Note that different extensions $h$ of the same function $\delta \cdot \tilde{f}(x): \tilde{X}_{+} \rightarrow$ $\mathbb{R}$ give equivalent norms on $L_{k, \delta}^{2}\left(Z_{+}, S^{ \pm}\right)$. Also note that the maps $L_{k}^{2}\left(Z_{+}, S^{ \pm}\right) \rightarrow L_{k, \delta}^{2}\left(Z_{+}, S^{ \pm}\right)$sending $\varphi$ to $e^{h} \varphi$ are isomorphisms.

5.2. End-periodic Dirac operators. Given an end-periodic manifold $Z_{+}$, with any choice of pair $(g, \beta)$ one associates the perturbed Dirac operator $D^{+}\left(Z_{+}, g, \beta\right): C^{\infty}\left(Z_{+}, S^{+}\right) \rightarrow C^{\infty}\left(Z_{+}, S^{-}\right)$. The closures of these operators with respect to the weighted Sobolev $L^{2}$-norms,

$$
D^{+}\left(Z_{+}, g, \beta\right): L_{1, \delta}^{2}\left(Z_{+}, S^{+}\right) \rightarrow L_{\delta}^{2}\left(Z_{+}, S^{-}\right),
$$

(compare with $(7)$ ), are related by the commutative diagram

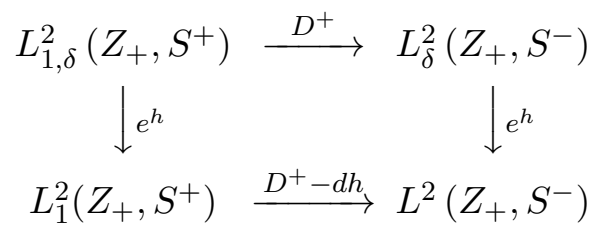

whose vertical arrows are isomorphisms. In particular, over the end $\tilde{X}_{+}$, the operators $D^{+}=D^{+}\left(\tilde{X}_{+}, g, \beta\right)$ and $D^{+}-d h=D^{+}\left(\tilde{X}_{+}, g, \beta\right)-$ $\delta f^{*}(d \theta)$ are intertwined by these isomorphisms.

Proposition 5.1. The operator (15) is Fredholm if and only if the operators $D_{\mu}^{+}(X, g, \beta)$ are invertible for all $\mu$ with $\operatorname{Re} \mu=\delta$.

Proof. According to Taubes [51, proposition 4.1], it is sufficient to show that the statement holds for the operator (11). The latter was the subject of Proposition 4.4.

q.e.d.

Corollary 5.2. Let $\left(g_{I}, \beta_{I}\right)$ be a regular path. For any $t \in I$, the operator $D^{+}\left(Z_{+}, g_{t}, \beta_{t}\right): L_{1, \delta}^{2}\left(Z_{+}, S^{+}\right) \rightarrow L_{\delta}^{2}\left(Z_{+}, S^{-}\right)$is Fredholm for all but a discrete set of $\delta \in \mathbb{R}$ with no accumulation points. This set may depend on $t$ but, for a fixed $t$, it is independent of the way the metric, perturbation 1-form, and the spin structure are extended to $Z$. 
For the rest of this section, we will assume that the pair $(g, \beta)$ belongs to a regular path even though it need not be regular itself. Let $\delta \in \mathbb{R}$ be such that the operator (15) is Fredholm, and denote its index by $\operatorname{ind}_{\delta} D^{+}\left(Z_{+}, g, \beta\right)$. Our study of this index will require the excision principle, which we will explain next.

5.3. The excision principle. The observation that the excision principle for operators on compact manifolds $[\mathbf{3}]$ (compare $[\mathbf{1 1}]$ ) extends to the non-compact setting is due to Gromov and Lawson [19]. A nice exposition of the non-compact version is in Charbonneau's thesis [8].

Let $A_{1}, B_{1}, A_{2}$, and $B_{2}$ be (not necessarily compact) oriented 4manifolds such that $\partial A_{1}=\partial A_{2}=Y$ and $\partial B_{1}=\partial B_{2}=-Y$, where $Y$ is a compact oriented 3-manifold. Let operators

$$
\begin{aligned}
& D_{1}: L^{2}\left(A_{1} \cup B_{1}\right) \rightarrow L^{2}\left(A_{1} \cup B_{1}\right) \\
& D_{2}: L^{2}\left(A_{2} \cup B_{2}\right) \rightarrow L^{2}\left(A_{2} \cup B_{2}\right)
\end{aligned}
$$

be (unbounded) Fredholm differential operators such that $D_{1}=D_{2}$ on $Y$. In our applications, $D_{1}$ and $D_{2}$ will be Dirac operators plus perhaps zero order terms. Suppose that

$$
\begin{aligned}
& \bar{D}_{1}: L^{2}\left(A_{1} \cup B_{2}\right) \rightarrow L^{2}\left(A_{1} \cup B_{2}\right) \\
& \bar{D}_{2}: L^{2}\left(A_{2} \cup B_{1}\right) \rightarrow L^{2}\left(A_{2} \cup B_{1}\right)
\end{aligned}
$$

defined as

$$
\bar{D}_{1}=\left\{\begin{array}{lll}
D_{1} & \text { on } & A_{1} \\
D_{2} & \text { on } & B_{2}
\end{array} \quad \text { and } \quad \bar{D}_{2}=\left\{\begin{array}{ccc}
D_{2} & \text { on } & A_{2} \\
D_{1} & \text { on } & B_{1}
\end{array}\right.\right.
$$

are (unbounded) Fredholm differential operators. Then

$$
\text { ind } D_{1}+\text { ind } D_{2}=\text { ind } \bar{D}_{1}+\text { ind } \bar{D}_{2} \text {. }
$$

5.4. Properties of the index. Our first application of the excision principle will be to show that the index of the operator (15) is well defined. A point that we will use without further comment is that Clifford multiplication by a real 1-form such as $d h$ is a skew-adjoint operator on spinors so that multiplication by a pure imaginary 1-form like $\beta$ is then self-adjoint.

Proposition 5.3. Let $\delta \in \mathbb{R}$ be such that the operator (15) is Fredholm. Then its index $\operatorname{ind}_{\delta} D^{+}\left(Z_{+}, g, \beta\right)$ is independent of the ways in which the metric, spin structure, perturbation form, and function $\delta \cdot \tilde{f}$ are extended to $Z$.

Proof. Consider two different extensions and apply the excision principle to the operators

$$
\begin{gathered}
D_{1}=D^{+}\left(Z_{+}, g_{1}\right)+\beta_{1}-d h_{1} \quad \text { on } \quad Z_{+}=Z \cup \tilde{X}_{+} \quad \text { and } \\
D_{2}=D^{+}\left(-Z_{+}, g_{2}\right)+\beta_{2}+d h_{2} \quad \text { on } \quad-Z_{+}=-\tilde{X}_{+} \cup(-Z),
\end{gathered}
$$


both of which are Fredholm. First, observe that

$$
\begin{aligned}
D^{+}\left(-Z_{+}, g_{2}\right)+\beta_{2}+d h_{2}=D^{-}\left(Z_{+}, g_{2}\right) & +\beta_{2}+d h_{2} \\
= & \left(D^{+}\left(Z_{+}, g_{2}\right)+\beta_{2}-d h_{2}\right)^{*} .
\end{aligned}
$$

Therefore, ind $D_{2}=-\operatorname{ind}_{\delta}\left(D^{+}\left(Z_{+}, g_{2}\right)+\beta_{2}\right)$. Second, the operator $\bar{D}_{1}$ is (up to zero order terms) the Dirac operator $D^{+}$on the compact manifold $Z \cup(-Z)$. In particular, ind $\bar{D}_{1}=0$. Finally, the manifold $-\tilde{X}_{+} \cup \tilde{X}_{+}$admits an orientation reversing involution that takes the operator $\bar{D}_{2}$ to its adjoint; therefore, ind $\bar{D}_{2}=0$. The excision principle now reads

$\operatorname{ind}_{\delta}\left(D^{+}\left(Z_{+}, g_{1}\right)+\beta_{1}\right)-\operatorname{ind}_{\delta}\left(D^{+}\left(Z_{+}, g_{2}\right)+\beta_{2}\right)=\operatorname{ind} \bar{D}_{1}+\operatorname{ind} \bar{D}_{2}=0$, which completes the proof.

q.e.d.

The next result will be helpful later when we compare the indices $\operatorname{ind}_{\delta} D^{+}\left(Z_{+}, g, \beta\right)$ for different values of $\delta$. Given an end-periodic manifold $Z_{+}=Z \cup \tilde{X}_{+}$, consider the end-periodic manifold $Z_{+}^{*}=Z \cup\left(-\tilde{X}_{-}\right)$, where

$$
\tilde{X}_{-}=\ldots \cup W_{-2} \cup W_{-1}=\tilde{X}-\tilde{X}_{+} .
$$

Note that this construction corresponds to the change of homology orientation on $X$. Respectively, the function $\tilde{f}$ is replaced by $-\tilde{f}$, and its extension $h: Z_{+} \rightarrow \mathbb{R}$ by $-h: Z_{+}^{*} \rightarrow \mathbb{R}$. Note, however, that $\beta$ and its extension are unchanged.

Proposition 5.4. The operator $D^{+}\left(Z_{+}, g, \beta\right)$ is Fredholm if and only if $D^{+}\left(Z_{+}^{*}, g, \beta\right)$ is Fredholm, and $\operatorname{ind}_{\delta} D^{+}\left(Z_{+}, g, \beta\right)=\operatorname{ind}_{\delta} D^{+}\left(Z_{+}^{*}, g, \beta\right)$.

Proof. Apply the excision principle to the operators $D_{1}=D^{+}\left(Z_{+}, g\right)$ $+\beta-d h$ and $D_{2}=D^{+}\left(-Z_{+}^{*}, g\right)+\beta-d h$, which are Fredholm. Since

$D^{+}\left(-Z_{+}^{*}, g\right)+\beta-d h=D^{-}\left(Z_{+}^{*}, g\right)+\beta-d h=\left(D^{+}\left(Z_{+}^{*}, g\right)+\beta+d h\right)^{*}$, we conclude that ind $D_{2}=-\operatorname{ind}_{\delta} D^{+}\left(Z_{+}^{*}, g, \beta\right)$. The excision principle then tells us that

$$
\operatorname{ind}_{\delta} D^{+}\left(Z_{+}, g, \beta\right)-\operatorname{ind}_{\delta} D^{+}\left(Z_{+}^{*}, g, \beta\right)=\text { ind } \bar{D}_{1}+\operatorname{ind} \bar{D}_{2} .
$$

The operator $\bar{D}_{1}$ is (up to zero order terms) the Dirac operator $D^{+}$ on the compact manifold $Z \cup(-Z)$, and hence ind $\bar{D}_{1}=0$. On the other hand, $\bar{D}_{2}=D^{+}(\tilde{X}, g)+\beta-\delta f^{*}(d \theta)$; hence ind $\bar{D}_{2}$ is equal to the index of

$$
D^{+}(\tilde{X}, g, \beta): L_{1, \delta}^{2}\left(\tilde{X}, S^{+}\right) \rightarrow L_{\delta}^{2}\left(\tilde{X}, S^{-}\right) .
$$

The latter index is zero, which can be seen as follows. Apply the Fourier-Laplace transform to the equation $D^{+}(\tilde{X}, g, \beta)(u)=0$ to obtain $D_{\mu}^{+}(X, g, \beta)\left(\hat{u}_{\mu}\right)=0$. Since all $D_{\mu}^{+}(X, g, \beta)$ with $\operatorname{Re} \mu=\delta$ are isomorphisms, we conclude that $\hat{u}_{\mu}=0$. Integrating over $I(\nu)$ with $\operatorname{Re} \nu=\delta$ gives $u=0$, hence ker $D^{+}(\tilde{X}, g, \beta)=0$. Similarly, coker $D^{+}(\tilde{X}, g, \beta)=$ 0 by the same argument applied to the operator $D^{-}(\tilde{X}, g, \beta)$. q.e.d. 


\section{The change of index formula}

This section is devoted to comparing the indices $\operatorname{ind}_{\delta} D^{+}\left(Z_{+}, g, \beta\right)$ for different values of $\delta \in \mathbb{R}$. We continue to assume that the pair $(g, \beta)$ belongs to a regular path even though it need not be regular itself. The resulting formula (20) contains as a special case the formula of $[\mathbf{2 8}$, theorem 1.2] (so in particular we provide a new and rather different proof of the latter). The indices ind $D^{+}\left(Z_{+}, g, \beta\right)$ for different $g$ and $\beta$ will be compared in the next section.

6.1. Reduction to an index problem on $\tilde{X}$. Given $\delta_{1}, \delta_{2} \in \mathbb{R}$, consider a smooth function $\delta: \tilde{X} \rightarrow[0,1]$ such that $\delta(x)=\delta_{1}$ on $W_{n}$ with $n \leq-1$ and $\delta(x)=\delta_{2}$ on $W_{n}$ with $n \geq 1$. Let $h(x)=\delta(x) \cdot \tilde{f}(x)$, and say that $\varphi \in L_{k ; \delta_{1}, \delta_{2}}^{2}\left(\tilde{X}, S^{ \pm}\right)$if and only if $e^{h} \varphi \in L_{k}^{2}\left(\tilde{X}, S^{ \pm}\right)$. In particular, if $\delta_{1}=\delta_{2}=\delta$, we get back the spaces $L_{k, \delta}^{2}\left(\tilde{X}, S^{ \pm}\right)$. As before, we have the commutative diagram

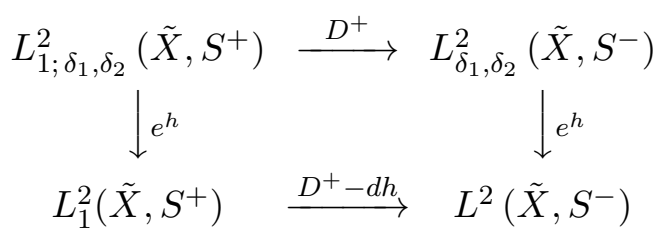

whose vertical arrows are isomorphisms, and we conclude that

$$
D^{+}=D^{+}(\tilde{X}, g, \beta): L_{1 ; \delta_{1}, \delta_{2}}^{2}\left(\tilde{X}, S^{+}\right) \rightarrow L_{\delta_{1}, \delta_{2}}^{2}\left(\tilde{X}, S^{-}\right)
$$

is a Fredholm operator for all $\delta_{1}, \delta_{2} \in \mathbb{R}$ away from a discrete set. We will denote its index by $\operatorname{ind}_{\delta_{1}, \delta_{2}} D^{+}(\tilde{X}, g, \beta)$.

Proposition 6.1. For any $\delta_{1}, \delta_{2} \in \mathbb{R}$ away from a certain discrete set with no accumulation points,

$$
\operatorname{ind}_{\delta_{2}} D^{+}\left(Z_{+}, g, \beta\right)-\operatorname{ind}_{\delta_{1}} D^{+}\left(Z_{+}, g, \beta\right)=\operatorname{ind}_{\delta_{1}, \delta_{2}} D^{+}(\tilde{X}, g, \beta) .
$$

Proof. This is seen by a repeated application of the excision principle, the functions $h_{1}, h_{2}: Z_{+} \rightarrow \mathbb{R}$ extending $\delta_{1} \cdot \tilde{f}(x)$ and $\delta_{2} \cdot \tilde{f}(x)$, respectively:

$$
\begin{aligned}
\operatorname{ind}_{\delta_{2}} D^{+}\left(Z_{+},\right. & g, \beta)-\operatorname{ind}_{\delta_{1}} D^{+}\left(Z_{+}, g, \beta\right) \\
& =\operatorname{ind}\left(D^{+}\left(Z_{+}, g, \beta\right)-d h_{2}\right)-\operatorname{ind}\left(D^{+}\left(Z_{+}, g, \beta\right)-d h_{1}\right) \\
& =\operatorname{ind}\left(D^{+}\left(Z_{+}, g, \beta\right)-d h_{2}\right)-\operatorname{ind}\left(D^{+}\left(Z_{+}^{*}, g, \beta\right)+d h_{1}\right) \\
& =\operatorname{ind}\left(D^{+}\left(Z_{+}, g, \beta\right)-d h_{2}\right)+\operatorname{ind}\left(D^{+}\left(-Z_{+}^{*}, g, \beta\right)-d h_{1}\right) \\
& =\operatorname{ind}\left(D^{+}(\tilde{X}, g, \beta)-d h\right)=\operatorname{ind}_{\delta_{1}, \delta_{2}} D^{+}(\tilde{X}, g, \beta) .
\end{aligned}
$$


6.2. Change of index via residues. Our next goal will be to compute the index $\operatorname{ind}_{\delta_{1}, \delta_{2}} D^{+}(\tilde{X}, g, \beta)$ in terms of the holomorphic family $D_{\mu}^{+}(X, g, \beta)=D^{+}(X, g, \beta)-\mu \cdot f^{*}(d \theta)$.

Fix a smooth function $\zeta: \tilde{X} \rightarrow \mathbb{R}$ such that $0 \leq \zeta \leq 1, \zeta=0$ on $W_{n}$ with $n \leq-1$, and $\zeta=1$ on $W_{n}$ with $n \geq 1$. Let $u \in L_{1 ; \delta_{1}, \delta_{2}}^{2}\left(\tilde{X}, S^{+}\right)$be a solution of the equation $D^{+}(\tilde{X}, g, \beta)(u)=0$. Write

$$
u=(1-\zeta) u+\zeta u=v+w,
$$

where $v=(1-\zeta) u \in L_{1, \delta_{1}}^{2}\left(\tilde{X}, S^{+}\right)$and $w=\zeta u \in L_{1, \delta_{2}}^{2}\left(\tilde{X}, S^{+}\right)$. A straightforward calculation shows that

$$
D^{+}(\tilde{X}, g, \beta)(v)=-k \quad \text { and } \quad D^{+}(\tilde{X}, g, \beta)(w)=k,
$$

where $k=d \zeta \cdot u$.

Since $k$ is supported in $W_{0}$, its Fourier-Laplace transform $\hat{k}_{\mu}$ is obviously holomorphic as a function of $\mu$ in the entire complex plane. Apply Lemma 4.3 to $w \in L_{1, \delta_{2}}^{2}\left(\tilde{X}, S^{+}\right)$supported in $\tilde{X}_{+}$to conclude that $\hat{w}_{\mu}$ is holomorphic in the half plane $\operatorname{Re} \mu<\delta_{2}$. A similar argument shows that $\hat{v}_{\mu}$ is holomorphic in the half plane $\operatorname{Re} \mu>\delta_{1}$. Hence the application of the Fourier-Laplace transform to the above two equations yields equations

$$
D_{\mu}^{+}(X, g, \beta)\left(\hat{v}_{\mu}\right)=-\hat{k}_{\mu} \quad \text { and } \quad D_{\mu}^{+}(X, g, \beta)\left(\hat{w}_{\mu}\right)=\hat{k}_{\mu},
$$

which hold in the half planes $\operatorname{Re} \mu>\delta_{1}$ and $\operatorname{Re} \mu<\delta_{2}$, respectively.

The inverse $R_{\mu}$ of the holomorphic family $D_{\mu}^{+}(X, g, \beta)$ is a meromorphic function of $\mu$ in the entire complex plane; see Theorem 4.6. Therefore, away from the poles of $R_{\mu}$, we have the equations

$$
\hat{v}_{\mu}=-R_{\mu} \hat{k}_{\mu} \quad \text { and } \quad \hat{w}_{\mu}=R_{\mu} \hat{k}_{\mu} .
$$

This allows us to extend $\hat{v}_{\mu}$ and $\hat{w}_{\mu}$ to meromorphic functions in the entire complex plane, called again $\hat{v}_{\mu}$ and $\hat{w}_{\mu}$. Since (by Proposition 4.1) the restriction of $\hat{v}_{\mu}$ to every interval $I\left(\delta_{1}+i \alpha\right)$ is square integrable, we conclude that $\hat{v}_{\mu}$ does not have poles on $\operatorname{Re} \mu=\delta_{1}$. Similarly, $\hat{w}_{\mu}$ does not have poles on $\operatorname{Re} \mu=\delta_{2}$.

The function $u=v+w$ with $v \in L_{1, \delta_{1}}^{2}\left(\tilde{X}, S^{+}\right)$and $w \in L_{1, \delta_{2}}^{2}\left(\tilde{X}, S^{+}\right)$ can now be recovered using the inverse Fourier-Laplace transform (see $(9))$ :

$$
\begin{aligned}
u(x+n)=\frac{1}{2 \pi i} \int_{I\left(\delta_{2}+i \alpha\right)} e^{-\mu(f(x)+n)} R_{\mu} \hat{k}_{\mu}(x) d \mu & \\
& -\frac{1}{2 \pi i} \int_{I\left(\delta_{1}+i \alpha\right)} e^{-\mu(f(x)+n)} R_{\mu} \hat{k}_{\mu}(x) d \mu .
\end{aligned}
$$


Let $\alpha$ be any real number such that $R_{\mu} \hat{k}_{\mu}(x)$ does not have poles on the horizontal lines $\operatorname{Im} \mu=\alpha$ and (consequently) $\operatorname{Im} \mu=\alpha+2 \pi$, and integrate $e^{-\mu(f(x)+n)} R_{\mu} \hat{k}_{\mu}(x)$ over the positively oriented contour $\Gamma$ shown in Figure 2.

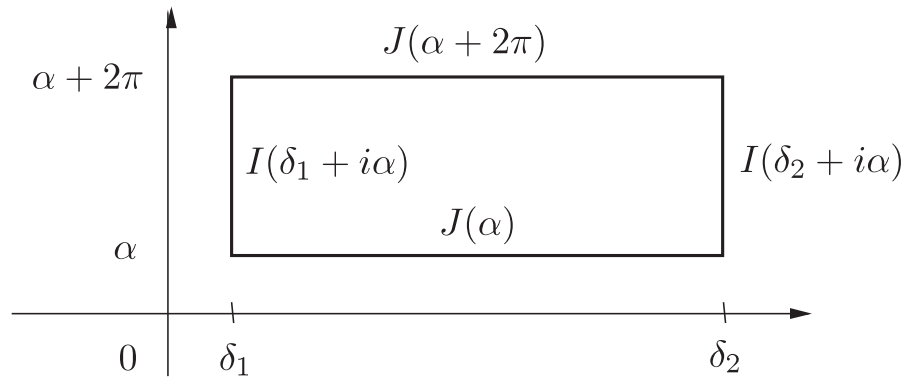

Figure 2. Contour $\Gamma$

Lemma 6.2. The contributions to the above contour integral from the sides $J(\alpha)$ and $J(\alpha+2 \pi)$ of $\Gamma$ cancel each other.

Proof. Let $\mu \in J(\alpha)$, then $\mu+2 \pi i \in J(\alpha+2 \pi)$ and $D_{\mu+2 \pi i}^{+}(X, g, \beta)=$ $D^{+}(X, g, \beta)-(\mu+2 \pi i) f^{*}(d \theta)=e^{2 \pi i f(x)}\left(D^{+}(X, g, \beta)-\mu f^{*}(d \theta)\right) e^{-2 \pi i f(x)}$. Therefore,

$$
R_{\mu+2 \pi i}=e^{2 \pi i f(x)} R_{\mu} e^{-2 \pi i f(x)} .
$$

Together with the fact that $\hat{k}_{\mu+2 \pi i}(x)=e^{2 \pi i f(x)} \hat{k}_{\mu}$ (see (8)), this implies that

$$
e^{-(\mu+2 \pi i)(f(x)+n)} R_{\mu+2 \pi i} \hat{k}_{\mu+2 \pi i}(x)=e^{-\mu(f(x)+n)} R_{\mu} \hat{k}_{\mu}(x),
$$

and the statement follows.

q.e.d.

A straightforward application of the Cauchy integral formula then leads us to the formula

$$
\begin{aligned}
u(x+n)=\frac{1}{2 \pi i} \oint_{\Gamma} e^{-\mu(f(x)+n)} R_{\mu} \hat{k}_{\mu}(x) d \mu & \\
= & \sum_{j} \operatorname{Res}_{\mu_{j}}\left(e^{-\mu(f(x)+n)} R_{\mu} \hat{k}_{\mu}(x)\right),
\end{aligned}
$$

where $\mu_{j}$ are the poles of $R_{\mu}$ inside the contour $\Gamma$. This formula describes the kernel of the operator (16). The residues will be explicitly calculated in the next section; note however that if $\delta_{1} \leq \delta_{2}$, then both functions $\hat{v}_{\mu}$ and $\hat{w}_{\mu}$ are holomorphic inside $\Gamma$, and hence the kernel of the operator (16) vanishes.

Next, the cokernel of $D^{+}(\tilde{X}, g, \beta)$ in the $L_{\delta_{1}, \delta_{2}}^{2}$ norm on $\tilde{X}$ is isomorphic to the kernel of $D^{-}(\tilde{X}, g, \beta)$ in the $L_{-\delta_{1},-\delta_{2}}^{2}$ norm, and hence can be calculated in terms of the residues as above. Again, this cokernel 
vanishes whenever $-\delta_{1} \leq-\delta_{2}$, that is, $\delta_{2} \leq \delta_{1}$. Therefore, depending on which of the weights $\delta_{1}$ or $\delta_{2}$ is larger, either the kernel or the cokernel of the operator (16) vanishes. We will assume without loss of generality that $\delta_{2} \leq \delta_{1}$, so that $\operatorname{ind}_{\delta_{1}, \delta_{2}} D^{+}(\tilde{X}, g, \beta)$ is equal to the dimension of the kernel of $D^{+}(\tilde{X}, g, \beta)$ described by the above residue formula.

6.3. Calculating the residues. Let $\mu_{j}$ be a pole of $R_{\mu}$, and write the Laurent series of $R_{\mu} \hat{k}_{\mu}(x)$ near $\mu_{j}$ in the form

$$
R_{\mu} \hat{k}_{\mu}(x)=\sum_{\ell=-m}^{\infty} b_{\ell}(x)\left(\mu-\mu_{j}\right)^{\ell}
$$

for some spinors $b_{\ell}(x)$. Apply the operator $D_{\mu}=D_{\mu}^{+}(X, g, \beta)$ to both sides of this equality to obtain

$$
\begin{aligned}
\hat{k}_{\mu}(x) & =\sum_{\ell} D_{\mu} b_{\ell}(x)\left(\mu-\mu_{j}\right)^{\ell} \\
& =\sum_{\ell} D_{\mu_{j}} b_{\ell}(x)\left(\mu-\mu_{j}\right)^{\ell}-\sum_{\ell} f^{*}(d \theta) b_{\ell}(x)\left(\mu-\mu_{j}\right)^{\ell+1} .
\end{aligned}
$$

The fact that $\hat{k}_{\mu}$ is an entire function then implies that the coefficients $b_{\ell}(x)$ solve the system

$$
\left\{\begin{array}{l}
D_{\mu_{j}} b_{-1}=f^{*}(d \theta) b_{-2} \\
\cdots \\
D_{\mu_{j}} b_{-m+1}=f^{*}(d \theta) b_{-m} \\
D_{\mu_{j}} b_{-m}=0
\end{array}\right.
$$

The spinors $b_{-1}, \ldots, b_{-m}$ determine the residues as follows. Write

$$
\begin{aligned}
& e^{-\mu(f(x)+n)} R_{\mu} \hat{k}_{\mu}(x)=e^{-\mu_{j}(f(x)+n)} e^{-\left(\mu-\mu_{j}\right)(f(x)+n)} R_{\mu} \hat{k}_{\mu}(x) \\
= & e^{-\mu_{j}(f(x)+n)} \sum_{k=0}^{\infty} \frac{(-1)^{k}}{k !}(f(x)+n)^{k}\left(\mu-\mu_{j}\right)^{k} \sum_{\ell=-m}^{\infty} b_{\ell}(x)\left(\mu-\mu_{j}\right)^{\ell},
\end{aligned}
$$

so that the residue of $e^{-\mu(f(x)+n)} R_{\mu} \hat{k}_{\mu}(x)$ at $\mu_{j}$ equals

$$
e^{-\mu_{j}(f(x)+n)} \sum_{p=1}^{m}(-1)^{p-1}(f(x)+n)^{p-1} b_{-p}(x) /(p-1) !
$$

Keeping in mind that $\tilde{f}(x)+n=\tilde{f}(x+n)$ for all $x \in W_{0}$ and all integers $n$, we can rewrite the latter formula as

$$
e^{-\mu_{j} \tilde{f}(x)} \sum_{p=1}^{m}(-1)^{p-1} \tilde{f}(x)^{p-1} b_{-p}(x) /(p-1) !
$$

Denote by $d\left(\mu_{j}\right)$ the number of linearly independent solutions of the equation $D^{+}(\tilde{X}, g, \beta)(u)=0$ of the form (18). Equivalently, $d\left(\mu_{j}\right)$ is 
the dimension of the vector space of solutions of system (17). Note that ker $D_{\mu_{j}}=0$ if and only if $d\left(\mu_{j}\right)=0$.

Remark 6.3. Expand the meromorphic function $R_{\mu}$ near its pole $\mu_{j}$ into Laurent series,

$$
R_{\mu}=\sum_{\ell=-m}^{\infty} A_{\ell}\left(\mu-\mu_{j}\right)^{\ell} .
$$

The equation $D_{\mu} R_{\mu}=I$ then implies that the operators $A_{\ell}$ solve the system

$$
\left\{\begin{array}{l}
D_{\mu_{j}} A_{-1}=f^{*}(d \theta) A_{-2} \\
\cdots \\
D_{\mu_{j}} A_{-m+1}=f^{*}(d \theta) A_{-m} \\
D_{\mu_{j}} A_{-m}=0
\end{array}\right.
$$

similar to (17). In particular, if $0 \neq b \in \operatorname{im} A_{-1}$ so that $b=A_{-1}(a)$, then setting $b_{-j}=A_{-j}(a)$ gives a solution of the system (17), with $b_{-1}=b$. Since $A_{-1}$ is in fact the operator $P_{\mu_{j}}$ defined in (14), we readily conclude that

$$
\operatorname{rank} P_{\mu_{j}} \leq d\left(\mu_{j}\right)
$$

6.4. Change of index formula. The proof of Lemma 6.2 tells us that the operators $D_{\mu+2 \pi i}^{+}(X, g, \beta)$ and $D_{\mu}^{+}(X, g, \beta)$ are isomorphic. We will use this fact to write

$$
D_{z}^{+}(X, g, \beta)=D^{+}(X, g, \beta)-\ln z \cdot f^{*}(d \theta)
$$

for $D_{\mu}^{+}(X, g, \beta)$ (compare with (5)) and also $d(z)$ for $d(\mu)$ and $P_{z}$ for $P_{\mu}$ if $z=e^{\mu}$. For any $\delta \leq \delta^{\prime}$ that make the operator (15) Fredholm, we have the following change of index formula:

$$
\operatorname{ind}_{\delta} D^{+}\left(Z_{+}, g, \beta\right)-\operatorname{ind}_{\delta^{\prime}} D^{+}\left(Z_{+}, g, \beta\right)=\sum_{e^{\delta}<|z|<e^{\delta^{\prime}}} d(z) .
$$

\section{The spectral flow formula}

In this section, we will describe how the index of the operator (7) changes along a special path $\left(g_{I}, \beta_{I}\right)$. The argument is strongly intertwined with the discussion of parameterized Seiberg-Witten moduli spaces $\mathcal{M}_{I}$ in Section 2. The change of index formula (see Theorem 7.3), which we refer to as the spectral flow formula, is much more precise than the formula (20) of the previous section. 
7.1. The reducibles. We begin by reviewing the transversality of the intersection $\mathcal{M}_{I}^{0}=\widetilde{\mathcal{M}}_{I} \cap \partial \mathcal{Z}_{I}$ for special paths $\left(g_{I}, \beta_{I}\right)$. Understanding this transversality in very concrete terms will be crucial for our discussion.

Let $\left(g_{I}, \beta_{I}\right)$ be a special path as in Theorem 2.5 that makes $\mathcal{M}_{I}$ regular and, in particular, $\mathcal{M}_{I}^{0}$ at most finite. Suppose $\tau \in I$ is such that $\mathcal{M}^{0}\left(X, g_{\tau}, \beta_{\tau}\right)$ is not empty. After a change of coordinates on $I$, we may assume that $\tau=0$ and that, for all $t$ sufficiently close to zero, $g_{t}$ is constant. We will use the notations $g_{0}=g$ and $\beta_{0}=\beta$.

Let $[0, A, 0, \varphi]$ be a point in $\mathcal{M}^{0}(X, g, \beta) \subset \mathcal{M}_{I}^{0}=\left(\partial \chi_{I}\right)^{-1}\left(d^{+} \beta_{I}\right)$. The fact that $\partial \chi_{I}$ is transversal to the section $d^{+} \beta_{I}$ means that the linearization of the map $\partial \chi_{I}-d^{+} \beta_{I}: \partial \mathcal{Z}_{I} \rightarrow \Omega_{+}^{2}(X, i \mathbb{R})$ at $[0, A, 0, \varphi]$ is a surjective linear operator. This operator will be called $\mathcal{D}$. The kernel of $\mathcal{D}$, which is necessarily zero dimensional, is then the tangent space to $\mathcal{M}_{I}^{0}$ at $[0, A, 0, \varphi]$

Using special paths allows us to avoid differentiating the metric, which provides for a particularly simple formula for $\mathcal{D}$. More precisely, the operator $\mathcal{D}$ in question is the operator

$$
\mathcal{D}: \mathbb{R} \times \Omega^{1}(X ; i \mathbb{R}) \times \Gamma\left(S^{+}\right)^{\perp} \longrightarrow \mathcal{H}^{0}(X, i \mathbb{R})^{\perp} \times \Omega_{+}^{2}(X, i \mathbb{R}) \times \Gamma\left(S^{-}\right)
$$

given by

$$
\mathcal{D}(v, b, \psi)=\left(-d^{*} b, d^{+} b-v d^{+} \dot{\beta}, D_{A}^{+}(X, g)(\psi)+b \cdot \varphi\right),
$$

compare with [23, lemma 27.1.1]. Here, $\Gamma\left(S^{+}\right)^{\perp}$ consists of all spinors $\psi$ such that $\langle\varphi, \psi\rangle_{L^{2}}=0$, and $\mathcal{H}^{0}(X ; i \mathbb{R})^{\perp} \subset \Omega^{0}(X, i \mathbb{R})$ consists of all functions $h: X \rightarrow i \mathbb{R}$ perpendicular to the subspace of constant functions $\mathcal{H}^{0}(X ; i \mathbb{R})=i \mathbb{R}$. The notation $\dot{\beta}$ means the derivative of $\beta_{t}$ with respect to $t$ evaluated at $t=0$.

Let us change variables $(v, b, \psi)$ to $(v, a, \psi)$ with $a=b-v \dot{\beta}$. The above operator then takes the form

$$
\mathcal{D}(v, a, \psi)=\left(-d^{*} a, d^{+} a, D_{A}^{+}(X, g)(\psi)+a \cdot \varphi+v \dot{\beta} \cdot \varphi\right)
$$

(remember that $d^{*} \beta_{t}=0$ for all $t$, hence $d^{*} \dot{\beta}=0$ ). In plain terms, the vanishing of ker $\mathcal{D}$ means that the following system of equations on $v$, $a$, and $\psi$ has a unique solution $(v, a, \psi)=(0,0,0)$ :

$$
\left\{\begin{array}{l}
d^{*} a=0, \\
d^{+} a=0, \\
D_{A}^{+}(X, g)(\psi)+a \cdot \varphi+v \dot{\beta} \cdot \varphi=0, \\
<\varphi, \psi>_{L^{2}}=0 .
\end{array}\right.
$$


7.2. Harmonic functions. Before we go on to deduce our spectral flow formula, we need to fix the function $f: X \rightarrow \mathbb{R}$ that was built into the definition of ind $D^{+}\left(Z_{+}, g, \beta\right)$ but has remained pretty much arbitrary until now. We will choose $f$ to be harmonic. The following existence and uniqueness result for harmonic functions can be found in Eells and Lemaire [13, section 7].

Lemma 7.1. For any metric $g$ on $X$, there exists a function $f: X \rightarrow$ $S^{1}$ that is harmonic with respect to $g$ and has the property that $f^{*}(d \theta)$ represents the generator $1 \in \mathbb{Z}=H^{1}(X ; \mathbb{Z})$. Moreover, such an $f$ is unique up to translation of $S^{1}$.

7.3. Change of index as spectral flow. Let $\left(g_{0}, \beta_{0}\right)$ and $\left(g_{1}, \beta_{1}\right)$ be two regular pairs of metrics and perturbations connected by a special path $\left(g_{I}, \beta_{I}\right)$ as in Theorem 2.5 so that $\mathcal{M}_{I}$ is regular and $g_{I}$ is constant near each $t \in I$ where $\mathcal{M}^{0}\left(X, g_{t}, \beta_{t}\right)$ is non-empty. Choose a smooth path of functions $f_{t}: X \rightarrow S^{1}$ harmonic with respect to $g_{t}$. Denote by $d_{t}(z)$ the dimension of the space of solutions of system (17) corresponding to the choice of metric $g_{t}$ and perturbation $\beta_{t}$.

Proposition 7.2. The set of pairs $(t, z)$ with $\operatorname{ker} D_{z}^{+}\left(X, g_{t}, \beta_{t}\right) \neq$ 0 and $|z|=1$ is in bijective correspondence with the points in $\mathcal{M}_{I}^{0}$. Moreover, for any $(t, z)$ in this set, $d_{t}(z)=\operatorname{rank} P_{z}=1$.

Proof. Let $\tau \in I$ be such that $\mathcal{M}^{0}\left(X, g_{\tau}, \beta_{\tau}\right) \subset \mathcal{M}_{I}^{0}$ is not empty. After changing coordinates, we will assume as in Section 7.1 that $\tau=0$ and write $g_{0}=g, f_{t}=f, d_{t}(z)=d(z)$, and $\beta_{0}=\beta$. A quadruple $[0, A, 0, \varphi]$ belongs to $\mathcal{M}^{0}(X, g, \beta)$ if and only if $F_{A}^{+}=d^{+} \beta, D_{A}^{+}(X, g)(\varphi)=0$, and $\|\varphi\|_{L^{2}}=1$. Up to gauge equivalence, $A=\beta-\ln z \cdot f^{*}(d \theta)$ for some $z \in \mathbb{C}$ with $|z|=1$. Therefore, $D_{z}^{+}(X, g, \beta)=D_{A}^{+}(X, g)$, and we have the claimed bijective correspondence.

We know from Proposition 2.6 that $\operatorname{dim}_{\mathbb{C}} \operatorname{ker} D_{z}^{+}(X, g, \beta)=1$, so that $d(z) \geq 1$. Suppose that $d(z)>1$. Then the last two equations of the system (17),

$$
\left\{\begin{array}{l}
D_{z}^{+}(X, g, \beta)\left(b_{-m+1}\right)=f^{*}(d \theta) \cdot b_{-m} \\
D_{z}^{+}(X, g, \beta)\left(b_{-m}\right)=0
\end{array}\right.
$$

have a solution with $b_{-m+1} \neq 0$ and $b_{-m} \neq 0$. Without loss of generality, we may assume that $\left\|b_{-m}\right\|_{L^{2}}=1$ and $<b_{-m}, b_{-m+1}>_{L^{2}}=0$. But then the triple $(v, a, \psi)=\left(0,-i f^{*}(d \theta), i b_{-m+1}\right)$ is a non-zero solution of the system (21) with $\varphi=b_{-m}$ and $A=\beta-\ln z \cdot f^{*}(d \theta)$, a contradiction. That $\operatorname{rank} P_{z}=1$ now follows from (19). q.e.d.

Let $D_{z}^{+}\left(X, g_{t}, \beta_{t}\right)$ be a family of perturbed Dirac operators as above and consider the subset

$$
\mathcal{S}_{I}=\left\{(t, z) \in[0,1] \times \mathbb{C}^{*} \mid \operatorname{ker} D_{z}^{+}\left(X, g_{t}, \beta_{t}\right) \neq 0\right\}
$$




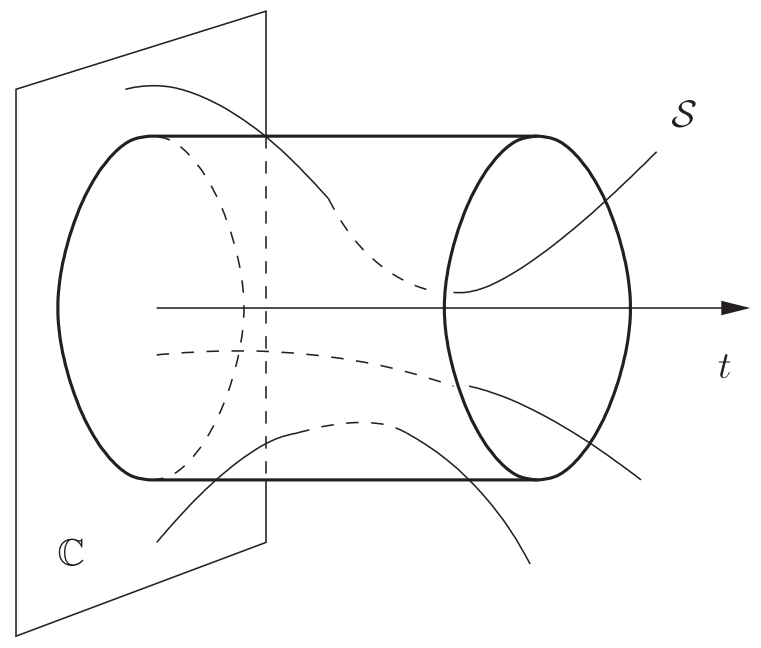

Figure 3. Spectral curves

of $[0,1] \times \mathbb{C}^{*}$. Its projection onto the second coordinate is in essence the parameterized spectral set $\Sigma_{I}$ defined in (13). According to Theorem 4.8 and Proposition 7.2, the set $\mathcal{S}_{I}$ is just a finite family of smooth curves near the cylinder $C=\left\{(t, z) \in[0,1] \times \mathbb{C}^{*}|| z \mid=1\right\}$. They will be referred to as the spectral curves. These curves intersect the cylinder $C$ in finitely many points $(t, z)$ with multiplicity one. We will show later in Lemma 8.4 that these intersections are in fact transversal.

The spectral flow $\operatorname{SF}\left(D_{z}^{+}\left(X, g_{I}, \beta_{I}\right)\right)$ along a special path $\left(g_{I}, \beta_{I}\right)$ is defined by counting the intersection points $\mathcal{S}_{I} \cap C$ with sign -1 if the spectral curve is entering the cylinder $C$, and +1 if it is leaving.

Theorem 7.3. Let $\beta_{0}, \beta_{1} \in \mathcal{P}$ be such that the pairs $\left(g_{0}, \beta_{0}\right)$ and $\left(g_{1}, \beta_{1}\right)$ are regular. Then the operators

$$
D^{+}\left(Z_{+}, g_{0}, \beta_{0}\right), D^{+}\left(Z_{+}, g_{1}, \beta_{1}\right): L_{1}^{2}\left(Z_{+}, S^{+}\right) \rightarrow L^{2}\left(Z_{+}, S^{-}\right)
$$

are Fredholm on any periodic end manifold $Z_{+}$whose end is modeled on $\tilde{X}$, and their indices differ by $\operatorname{SF}\left(D_{z}^{+}\left(X, g_{I}, \beta_{I}\right)\right)$ for any special path of metrics and perturbations.

Proof. Since $\mathcal{M}^{0}\left(X, g_{0}, \beta_{0}\right)$ and $\mathcal{M}^{0}\left(X, g_{1}, \beta_{1}\right)$ are both empty, the families $D_{z}^{+}\left(X, g_{0}, \beta_{0}\right)$ and $D_{z}^{+}\left(X, g_{1}, \beta_{1}\right)$ have zero kernels on the unit circle $|z|=1$ by Proposition 7.2. The operators $D^{+}\left(Z_{+}, g_{0}, \beta_{0}\right)$ and $D^{+}\left(Z_{+}, g_{1}, \beta_{1}\right)$ are then Fredholm by Proposition 5.1 .

Suppose that $\tau \in(0,1)$ is such that not all operators $D_{z}^{+}\left(X, g_{\tau}, \beta_{\tau}\right)$ with $|z|=1$ have zero kernel. After changing coordinates, we will assume that $\tau=0$. Let $z_{j}(j=1, \ldots, m)$ be all the points in the complex plane such that $\left|z_{j}\right|=1$ and $d_{0}\left(z_{j}\right)=1$. 
Choose $\varepsilon_{0}>0$ so that the portions of all the spectral curves through $\left(0, z_{j}\right), j=1, \ldots, m$, cut out by the condition $0<|t| \leq \varepsilon_{0}$, do not intersect the cylinder $C$ and have the property that $d_{t}(z) \leq 1$. By continuity of spectral curves, for any small $\delta>0$, one can find $\varepsilon>0$ such that $\varepsilon<$ $\varepsilon_{0}$ and the intersection of $\mathcal{S}_{I}$ with the cylinder $\left\{(t, z)|| t|\leq \varepsilon| z \mid,=e^{\delta}\right\}$ is empty. Then we have well-defined indices $\operatorname{ind}_{\delta} D^{+}\left(Z_{+}, g_{t}, \beta_{t}\right)$ for all $t \in[-\varepsilon, \varepsilon]$ and, by continuity of the index,

$$
\operatorname{ind}_{\delta} D^{+}\left(Z_{+}, g_{-\varepsilon}, \beta_{-\varepsilon}\right)=\operatorname{ind}_{\delta} D^{+}\left(Z_{+}, g_{\varepsilon}, \beta_{\varepsilon}\right) \text {. }
$$

On the other hand, equation (20) provides us with the formulas

$$
\left(\text { ind }-\operatorname{ind}_{\delta}\right)\left(D^{+}\left(Z_{+}, g_{-\varepsilon}, \beta_{-\varepsilon}\right)\right)=\sum_{1<|z|<e^{\delta}} d_{-\varepsilon}(z)
$$

and

$$
\left(\text { ind }-\operatorname{ind}_{\delta}\right)\left(D^{+}\left(Z_{+}, g_{\varepsilon}, \beta_{\varepsilon}\right)\right)=\sum_{1<|z|<e^{\delta}} d_{\varepsilon}(z)
$$

where $d_{ \pm \varepsilon}(z)$ is zero, except at finitely many points where it is one. Combining the three formulas above, we obtain

$$
\text { ind } \begin{aligned}
D^{+}\left(Z_{+}, g_{\varepsilon}, \beta_{\varepsilon}\right)-\text { ind } D^{+}\left(Z_{+},\right. & \left.g_{-\varepsilon}, \beta_{-\varepsilon}\right) \\
& =\sum_{1<|z|<e^{\delta}} d_{\varepsilon}(z)-\sum_{1<|z|<e^{\delta}} d_{-\varepsilon}(z) .
\end{aligned}
$$

One can further observe that, if $d_{-\varepsilon}(z)=1$, then $(-\varepsilon, z)$ belongs to the same component of $\mathcal{S}_{I}$ as $\left(0, z_{j}\right)$ for some $j$; the latter contributes -1 to the spectral flow. Similarly, if $d_{\varepsilon}(z)=1$, then $(\varepsilon, z)$ belongs to the same component of $\mathcal{S}_{I}$ at $\left(0, z_{k}\right)$ for some $k$; the latter contributes +1 to the spectral flow.

q.e.d.

\section{The invariant}

In this section, we will define the invariant $\lambda_{\mathrm{SW}}(X)$ and prove the first statement of Theorem A regarding it.

8.1. The invariant. Let $g$ be an arbitrary metric on $X$ and choose a regular pair $(g, \beta)$ of metric and perturbation. Define

$$
\lambda_{\mathrm{SW}}(X)=\# \mathcal{M}(X, g, \beta)-w(X, g, \beta) .
$$

Theorem 8.1. $\lambda_{\mathrm{SW}}(X)$ is independent of the choice of regular pair $(g, \beta)$.

Proof. Given two regular pairs, $\left(g_{0}, \beta_{0}\right)$ and $\left(g_{1}, \beta_{1}\right)$, choose a special path $\left(g_{I}, \beta_{I}\right)$ as in Theorem 2.5 so that the parameterized moduli space $\mathcal{M}_{I}$ is regular and the metric $g_{t}$ is constant near every value of $t \in I$ where $\mathcal{M}^{0}\left(X, g_{t}, \beta_{t}\right)$ is non-empty. According to Theorem 2.4,

$$
\# \mathcal{M}\left(X, g_{1}, \beta_{1}\right)-\# \mathcal{M}\left(X, g_{0}, \beta_{0}\right)=\# \mathcal{M}_{I}^{0} \text {, }
$$


where $\# \mathcal{M}_{I}^{0}$ stands for the signed count of points in $\mathcal{M}_{I}^{0}$. On the other hand, Theorem 7.3 tells us that

$$
w\left(X, g_{1}, \beta_{1}\right)-w\left(X, g_{0}, \beta_{0}\right)=\operatorname{SF}\left(D_{z}^{+}\left(X, g_{I}, \beta_{I}\right)\right),
$$

a signed count of points on $\mathcal{S}_{I} \cap C$. According to Proposition 7.2, the points in $\mathcal{M}_{I}^{0}$ and in $\mathcal{S}_{I} \cap C$ are in a bijective correspondence. That the corresponding points in $\mathcal{M}_{I}^{0}$ and $\mathcal{S}_{I} \cap C$ are counted with the same sign is proved in the following section.

q.e.d.

8.2. Comparing signs. We continue with the calculation that we started in Section 7.1. To figure out the orientation of $[0, A, 0, \varphi] \in \mathcal{M}_{I}^{0}$, consider the path of Fredholm operators

$$
\mathcal{D}_{u}(v, a, \psi)=\left(-d^{*} a, d^{+} a, D_{A}^{+}(X, g)(\psi)+u a \cdot \varphi+u v \dot{\beta} \cdot \varphi\right)
$$

parameterized by $u \in[0,1]$. It connects the operator $\mathcal{D}_{0}=\left(-d^{*} \oplus d^{+}\right) \oplus$ $D_{A}^{+}(X, g)$ to our operator $\mathcal{D}=\mathcal{D}_{1}$. We will compute the orientation of $[0, A, 0, \varphi]$ by calculating the orientation transport along $\mathcal{D}_{u}$ using the formula (1.5.9) from [37]. That formula expresses the orientation transport as the product

$$
\operatorname{sign} \operatorname{det}\left(R_{0}\right) \cdot \operatorname{sign} \operatorname{det}\left(R_{1}\right) \cdot \prod_{u \in[0,1)}(-1)^{\operatorname{dim} \operatorname{ker} \mathcal{D}_{u}},
$$

where $R_{u}: \operatorname{ker} \mathcal{D}_{u} \rightarrow$ coker $\mathcal{D}_{u}, u=0,1$, are resonance operators defined as the derivative $(d / d u)\left(\mathcal{D}_{u}-\mathcal{D}_{0}\right)$ evaluated at $u=0,1$, followed by the $L^{2}$ orthogonal projection $\pi$ onto coker $\mathcal{D}_{u}$.

First we observe that, for any $u \neq 0$, the operator $\mathcal{D}_{u}$ has zero kernel, because any non-zero solution $(v, a, \psi)$ of the system

$$
\left\{\begin{array}{l}
d^{*} a=0 \\
d^{+} a=0 \\
D_{A}^{+}(X, g)(\psi)+u a \cdot \varphi+u v \dot{\beta} \cdot \varphi=0 \\
<\varphi, \psi>_{L^{2}}=0
\end{array}\right.
$$

would give a non-zero solution $(v, a, \psi / u)$ of system (21). Since ker $\mathcal{D}_{0}$ is even dimensional, the orientation transport is simply the sign of the determinant of the resonance operator $R_{0}: \operatorname{ker} \mathcal{D}_{0} \rightarrow$ coker $\mathcal{D}_{0}$. Of course, both ker $\mathcal{D}_{0}$ and coker $\mathcal{D}_{0}$ need to be oriented.

A straightforward calculation shows that $\operatorname{ker} \mathcal{D}_{0}=\mathbb{R} \oplus \mathcal{H}^{1}(X ; i \mathbb{R}) \cong$ $\mathbb{R}^{2}$ and coker $\mathcal{D}_{0}=$ coker $D_{A}^{+}(X, g)=\mathbb{C}$. They are canonically oriented, the former by the choice of homology orientation and the latter by the complex structure on coker $D_{A}^{+}(X, g)$.

Fix an isomorphism $\mathbb{R}^{2}=\operatorname{ker} \mathcal{D}_{0}$ sending $(v, c) \in \mathbb{R}^{2}$ to $\left(v, i c f^{*}(d \theta), 0\right)$ $\in$ ker $\mathcal{D}_{0}$ (remember that $f: X \rightarrow \mathbb{R}$ was chosen to be harmonic). Since $\left(\mathcal{D}_{u}-\mathcal{D}_{0}\right)(v, a, \psi)=(0,0, u a \cdot \varphi+u v \dot{\beta} \cdot \varphi)$, we conclude that the resonance operator $R_{0}: \mathbb{R}^{2} \rightarrow \operatorname{coker} D_{A}^{+}$can be written as

$$
R_{0}(v, c)=\pi\left(i c f^{*}(d \theta) \cdot \varphi+v \dot{\beta} \cdot \varphi\right) .
$$


Lemma 8.2. $\pi\left(i f^{*}(d \theta) \cdot \varphi\right) \neq 0$.

Proof. Suppose that, on the contrary, $\pi\left(i f^{*}(d \theta) \cdot \varphi\right)=0$. Then there is $\psi$ such that $i f^{*}(d \theta) \cdot \varphi=D_{A}^{+}(X, g)(\psi)$. Since $D_{A}^{+}(X, g)(\varphi)=0$, we may assume without loss of generality that $\langle\varphi, \psi\rangle_{L^{2}}=0$. But then $(v, a, \psi)=\left(0,-i f^{*}(d \theta), \psi\right)$ is a non-zero solution of the system (21), a contradiction.

q.e.d.

Corollary 8.3. The spinors $\pi\left(f^{*}(d \theta) \cdot \varphi\right)$ and $\pi\left(i f^{*}(d \theta) \cdot \varphi\right)=i \pi$ $\left(f^{*}(d \theta) \cdot \varphi\right)$ form a positively oriented basis in coker $D_{A}^{+}(X, g)=\mathbb{C}$.

Let us next study $\pi(\dot{\beta} \cdot \varphi) \in$ coker $D_{A}^{+}(X, g)$. Up to gauge equivalence, $A=\beta-\ln z \cdot f^{*}(d \theta)$ for some $z \in \mathbb{C}$ with $|z|=1$ so that $D_{A}^{+}(X, g)=$ $D_{z}^{+}(X, g, \beta)$, and the unit spinor $\varphi$ spans $\operatorname{ker} D_{z}^{+}(X, g, \beta)=\mathbb{C}$. Let $\left(t, z_{t}\right)$ be the spectral curve through $(0, z)$; it is a smooth curve if $t$ stays sufficiently close to 0 . Write $\ln z_{t}=a_{t}+i c_{t}$ so that $\ln z=a_{0}+i c_{0}=i c$, and consider a path of unit spinors $\varphi_{t}$ such that $\varphi_{0}=\varphi$ and

$$
\varphi_{t} \in \operatorname{ker}\left(D^{+}(X, g)-\left(a_{t}+i c_{t}\right) f^{*}(d \theta)+\beta_{t}\right) .
$$

Differentiate the equation

$$
\left(D^{+}(X, g)-\left(a_{t}+i c_{t}\right) f^{*}(d \theta)+\beta_{t}\right)\left(\varphi_{t}\right)=0
$$

with respect to $t$ at $t=0$. Since $\dot{D}^{+}(X, g)=0$, we obtain $D^{+}(X, g)(\dot{\varphi})+\left(-(\dot{a}+i \dot{c}) f^{*}(d \theta) \cdot \varphi+\dot{\beta} \cdot \varphi\right)+\left(-i c f^{*}(d \theta)+\beta\right) \cdot \dot{\varphi}=0$, or, equivalently,

$$
D_{A}^{+}(X, g)(\dot{\varphi})=(\dot{a}+i \dot{c}) f^{*}(d \theta) \cdot \varphi-\dot{\beta} \cdot \varphi .
$$

Projecting onto coker $D_{A}^{+}(X, g)$ (along the image of $D_{A}^{+}(X, g)$ ), we obtain

$$
\begin{aligned}
\pi(\dot{\beta} \cdot \varphi) & =\pi\left((\dot{a}+i \dot{c}) f^{*}(d \theta) \cdot \varphi\right) \\
& \left.=\dot{a} \pi\left(f^{*}(d \theta) \cdot \varphi\right)\right)+\dot{c} \pi\left(i f^{*}(d \theta) \cdot \varphi\right) .
\end{aligned}
$$

Therefore, with respect to the basis of coker $D_{A}^{+}(X, g)$ given by Corollary 8.3, the resonance operator $R_{0}$ has the matrix

$$
R_{0}=\left(\begin{array}{cc}
\dot{a} & 0 \\
\dot{c} & 1
\end{array}\right) .
$$

Lemma 8.4. The operator $R_{0}$ is non-singular, that is, $\operatorname{det} R_{0}=\dot{a} \neq$ 0 .

Proof. Suppose on the contrary that $\dot{a}=0$. Let $\psi=\dot{\varphi}+\alpha \varphi$ and choose $\alpha \in \mathbb{C}$ so that $\langle\varphi, \psi\rangle_{L^{2}}=0$. Then (25) implies that $(v, a, \psi)=\left(1,-i \dot{c} f^{*}(d \theta), \psi\right)$ is a non-zero solution of the system (21), a contradiction.

q.e.d.

A straightforward application of the orientation transport formula (22) gives us the following result. 
Corollary 8.5. The point $[0, A, 0, \varphi] \in \mathcal{M}^{0}(X, g, \beta)$ is oriented by $\operatorname{sign}(\dot{a})$.

This concludes the proof of Theorem 8.1 , because $\operatorname{sign}(\dot{a})= \pm 1$ is precisely the contribution of the point $(0, z) \in \mathcal{S}_{I} \cap C$ corresponding to $[0, A, 0, \varphi] \in \mathcal{M}_{I}^{0}$ to the spectral flow.

\section{Relation with the Rohlin invariant}

Let $X$ be an oriented spin smooth homology $S^{1} \times S^{3}$ with a fixed homology orientation, that is, a generator $1 \in H^{1}(X ; \mathbb{Z})$. Choose a connected 3-manifold $Y \subset X$ dual to this generator. Note that $Y$ is canonically oriented, and inherits a spin structure from $X$. The Rohlin invariant of $X$ is defined as $\rho(X)=\operatorname{sign}(Z) / 8(\bmod 2)$, where $Z$ is any smooth compact spin manifold with spin boundary $\partial Z=Y$; see $[\mathbf{4 2}$, 43, 48]. We will show that

$$
\lambda_{\mathrm{SW}}(X)=\# \mathcal{M}(X, g, \beta)-\operatorname{ind}_{\mathbb{C}} D^{+}\left(Z_{+}, g, \beta\right)-\operatorname{sign}(Z) / 8
$$

reduces mod 2 to the Rohlin invariant by arguing that first, $\# \mathcal{M}(X, g, \beta)$ is even because $\mathcal{M}(X, g, \beta)$ has quaternionic structure, and second, that ind $\mathbb{C}_{\mathbb{C}} D^{+}\left(Z_{+}, g, \beta\right)$ is even because $D^{+}\left(Z_{+}, g, \beta\right)$ is quaternionic linear. Neither is actually true unless we take special care of choosing proper metrics and perturbations, as described below.

9.1. Generic metrics. Let $Z_{+}=Z \cup \tilde{X}_{+}$be an end-periodic manifold whose end is modeled on the infinite cyclic cover of $X$. For any choice of metric $g$ on $X$, the Dirac operator $D^{+}\left(Z_{+}, g\right): L_{1}^{2}\left(Z_{+}, S^{+}\right) \rightarrow$ $L^{2}\left(Z_{+}, S^{-}\right)$is quaternionic linear, hence its index ind $\mathbb{C}_{\mathbb{C}} D^{+}\left(Z_{+}, g\right)$ is even - assuming of course that $D^{+}\left(Z_{+}, g\right)$ is Fredholm. So far we know two ways of ensuring Fredholmness. We can use Corollary 5.2 to conclude that $D^{+}\left(Z_{+}, g\right): L_{1, \delta}^{2}\left(Z_{+}, S^{+}\right) \rightarrow L_{\delta}^{2}\left(Z_{+}, S^{-}\right)$is Fredholm for all but a discrete set of $\delta \in \mathbb{R}$, or we can combine Propositions 2.2 and 7.2 to conclude that $D^{+}(X, g, \beta): L_{1}^{2}\left(Z_{+}, S^{+}\right) \rightarrow L^{2}\left(Z_{+}, S^{-}\right)$is Fredholm for generic $\beta \in \mathcal{P}$. However, introducing either a weight $\delta \neq 0$ or a perturbation $\beta \neq 0$ ruins the quaternionic linearity of the Dirac operator. The paper $[44]$ of the second and third authors provides a better way of achieving Fredholmness by perturbing the metric alone and hence preserving the quaternionic linearity.

Theorem 9.1. The operator $D^{+}\left(Z_{+}, g\right): L_{1}^{2}\left(Z_{+}, S^{+}\right) \rightarrow L^{2}\left(Z_{+}, S^{-}\right)$ is Fredholm for a generic choice of metric $g$ on $X$.

Note that a choice of metric $g$ as in the above theorem only guarantees (via Proposition 7.2) that $\mathcal{M}(X, g, 0)$ has no reducibles but not that it is regular. A further perturbation $\beta \in \mathcal{P}$ may be needed to ensure its regularity. If that perturbation is small enough, it will not create any 
reducibles in $\mathcal{M}(X, g, \beta)$. Moreover, according to Theorem 7.3 , we will have

$$
\operatorname{ind}_{\mathbb{C}} D^{+}\left(Z_{+}, g, \beta\right)=\operatorname{ind}_{\mathbb{C}} D^{+}\left(Z_{+}, g\right),
$$

meaning that the index ind $\mathbb{C}_{\mathbb{C}} D^{+}\left(Z_{+}, g, \beta\right)$ will be even despite the fact that $D^{+}\left(Z_{+}, g, \beta\right)$ may no longer be quaternionic linear.

Corollary 9.2. The index ind $\mathbb{C} D^{+}\left(Z_{+}, g, \beta\right)$ is even for a generic metric $g$ and a generic sufficiently small perturbation $\beta \in \mathcal{P}$.

9.2. $J$-action. The quaternionic structures on $S^{ \pm}$lead to a natural $\mathbb{Z} / 4$-action on the triples $(A, s, \varphi)$ given by $J(A, s, \varphi)=(-A, s, j \varphi)$. It is free because of the condition $\|\varphi\|_{L^{2}}=1$, and it descends to a free involution $J: \widetilde{\mathcal{Z}} \rightarrow \widetilde{\mathcal{Z}}$. The following result is straightforward, once we observe that $\tau(j \varphi)=-\tau(\varphi)$ in $(1)$.

Lemma 9.3. The map $\chi: \widetilde{\mathcal{Z}} \rightarrow \Omega_{+}^{2}(X, i \mathbb{R})$ is equivariant with respect to $J$ in that the following diagram commutes:

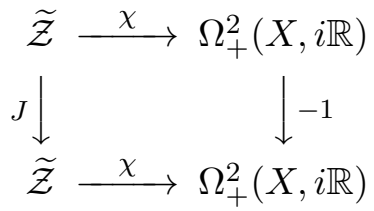

In particular, we see that $J$ does not act on $\mathcal{M}(X, g, \beta)$ unless $\beta=0$. Therefore, if we want to show that $\# \mathcal{M}(X, g, \beta)$ is even for a generic metric, we will need more elaborate perturbations. In what follows, we adopt the approach of $[\mathbf{3 2}]$.

Let us view $\chi$ as a section of the trivial bundle $\mathcal{E}=\widetilde{\mathcal{Z}} \times \Omega_{+}^{2}(X, i \mathbb{R}) \rightarrow$ $\widetilde{\mathcal{Z}}$ given by $\chi([A, s, \varphi])=\left([A, s, \varphi], F_{A}^{+}-s^{2} \tau(\varphi)\right)$. It is equivariant, meaning that the following diagram commutes:

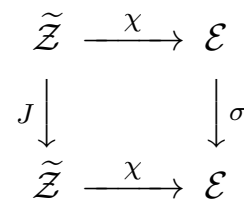

Here, $\sigma$ acts as $J$ on the base $\widetilde{\mathcal{Z}}$ and as -1 on each of the fibers of $\mathcal{E}$. Taking quotient by the free action of $J$ and $\sigma$, we obtain a section

$$
\chi^{\prime}: \widetilde{\mathcal{Z}}^{\prime} \rightarrow \mathcal{E}^{\prime}
$$

of the bundle $\mathcal{E}^{\prime}=\widetilde{\mathcal{Z}} \times{ }_{\sigma} \Omega_{+}^{2}(X, i \mathbb{R})$ over the Hilbert manifold $\widetilde{\mathcal{Z}}^{\prime}=\widetilde{\mathcal{Z}} / J$.

Adding a small generic section $\zeta^{\prime}: \widetilde{\mathcal{Z}}^{\prime} \rightarrow \mathcal{E}^{\prime}$ makes $\chi^{\prime}+\zeta^{\prime}$ transversal to the zero section of $\mathcal{E}^{\prime}$, and also makes its lift $\chi+\zeta: \widetilde{\mathcal{Z}} \rightarrow \mathcal{E}$ an equivariant section transversal to the zero section of $\mathcal{E}$. In fact, we can choose $\zeta=d^{+} \gamma$ for a map $\gamma: \widetilde{\mathcal{Z}} \rightarrow \mathcal{P}$ which is equivariant in that $\gamma([-A, s, j \varphi])=-\gamma([A, s, \varphi])$. The perturbations $\beta \in \mathcal{P}$ that we used 
before can then be viewed as constant maps $\beta: \widetilde{\mathcal{Z}} \rightarrow \mathcal{P}$. The perturbed Seiberg-Witten moduli space

$$
\mathcal{M}(X, g, \gamma)=\left(\chi+d^{+} \gamma\right)^{-1}(0)
$$

is a compact regular manifold of dimension zero acted upon freely by the involution $J$, cf. [32]. In particular, $\mathcal{M}(X, g, \gamma)$ consists of an even number of points.

Proposition 9.4. For a generic metric $g$ and sufficiently small generic perturbations $\beta$ and $\gamma$ as above, the moduli spaces $\mathcal{M}(X, g, \beta)$ and $\mathcal{M}(X, g, \gamma)$ are in bijective correspondence. In particular, the number $\# \mathcal{M}(X, g, \beta)$ is even.

Proof. In the Hilbert space of all perturbations $\widetilde{\mathcal{Z}} \rightarrow \mathcal{P}$, the zero perturbation corresponds to the moduli space $\mathcal{M}(X, g, 0)$ with empty $\mathcal{M}^{0}(X, g, 0)$ by our choice of generic metric $g$. By continuity, there is a small ball in this space centered at 0 such that all the perturbations $\eta$ in it have the property that $\mathcal{M}^{0}(X, g, \eta)$ is empty. Choose $\beta$ and $\gamma$ sufficiently small so that they belong to this ball, and connect $\beta$ to $\gamma$ by the path $\eta_{t}=(1-t) \beta+t \gamma, 0 \leq t \leq 1$. Along this path, all $\mathcal{M}\left(X, g, \eta_{t}\right)$ have empty $\mathcal{M}^{0}\left(X, g, \eta_{t}\right)$ but are not necessarily regular, except at the endpoints. Perturb this path a little rel its endpoints into $\eta_{t}^{\prime}$ so that $\eta_{t}^{\prime}$ stays inside the ball, while keeping all $\mathcal{M}^{0}\left(X, g, \eta_{t}^{\prime}\right)$ empty and making $\mathcal{M}\left(X, g, \eta_{t}^{\prime}\right)$ regular. The parameterized moduli space

$$
\bigcup_{t \in[0,1]}\{t\} \times \mathcal{M}\left(X, g, \eta_{t}^{\prime}\right)
$$

provides an oriented cobordism between $\mathcal{M}(X, g, \beta)$ and $\mathcal{M}(X, g, \gamma)$, and the result follows. q.e.d.

\section{Negative-definite manifolds with $b_{1}=1$}

The definition of the invariant $\lambda_{\mathrm{SW}}(X)$ extends to a more general topological setup, encountered $[\mathbf{3 9}, \mathbf{5 2}]$ in the study of non-Kähler complex surfaces, particularly those of type $\mathrm{VII}_{0}$. We will present two sets of hypotheses that yield a well-defined invariant; unfortunately, both are stronger than the minimal hypothesis that $H_{1}(X)=\mathbb{Z}$ and $b_{+}^{2}(X)=0$ one would hope for.

In the first situation, we assume that $X$ is a smooth oriented closed 4-manifold and that the following conditions hold:

$$
\begin{aligned}
& H_{1}(X ; \mathbb{Z})=\mathbb{Z}, b_{+}^{2}(X)=0, b_{-}^{2}(X)=n, \text { and } \\
& H_{3}(X) \text { is generated by an integral homology sphere } Y \subset X .
\end{aligned}
$$

In the second situation, we assume that

$$
H_{1}(X ; \mathbb{Z})=\mathbb{Z}, b_{+}^{2}(X)=0, \text { and } b_{-}^{2}(X)=1
$$


but no longer make any special hypothesis about the topology of a 3manifold $Y$ generating $H_{3}(X)$.

By Donaldson's theorem, the intersection form of $X$ is diagonalizable over the integers. Let $\mathfrak{s}_{X}$ be a $\operatorname{Spin}^{c}$ structure with $c_{1}\left(\mathfrak{s}_{X}\right)$ dual to the sum of the vectors in a diagonalizing basis, so that

$$
c_{1}\left(\mathfrak{s}_{X}\right)^{2}=\operatorname{sign}(X)=-n .
$$

Note that $H^{2}(X ; \mathbb{Z})$ is torsion-free; hence specifying $c_{1}\left(\mathfrak{s}_{X}\right)$ actually determines $\mathfrak{s}_{X}$. Any two bases diagonalizing $H_{2}(X ; \mathbb{Z})$ differ by permutation and change of signs of the basis elements, so there are in principle $2^{n-1}$ choices of $\operatorname{spin}^{c}$ structure to consider when defining our invariant. (The 'charge conjugation' of the Seiberg-Witten equations [31, section 6.8] implies that the invariant is preserved when one reverses all of the signs of the basis elements.) If $X$ is an $n$-fold blowup of a homology $S^{1} \times S^{3}$, then these all yield the same invariant up to sign, but we do not know if this holds in general.

For any choice of $\operatorname{spin}^{c}$ structure $\mathfrak{s}_{X}$ as above and any choice of metric $g$ on $X$, the index of the $\operatorname{spin}^{c}$ Dirac operator $D^{+}\left(X, \mathfrak{s}_{X}, g\right)$ is given by

$$
\left(c_{1}\left(\mathfrak{s}_{X}\right)^{2}-\operatorname{sign}(X)\right) / 8=0 .
$$

This implies that the Seiberg-Witten moduli space corresponding to $\mathfrak{s}_{X}$ has formal dimension 0. An analogue of Proposition 2.2 then shows that, for a generic perturbation $\beta \in \mathcal{P}$, the perturbed Seiberg-Witten moduli space $\mathcal{M}\left(X, \mathfrak{s}_{X}, g, \beta\right)$ is a compact zero-dimensional manifold with no reducibles. Choose a homology orientation on $X$, that is, a generator $1 \in H^{1}(X ; \mathbb{Z})$; then $\mathcal{M}\left(X, \mathfrak{s}_{X}, g, \beta\right)$ is canonically oriented, and we denote by $\# \mathcal{M}\left(X, \mathfrak{s}_{X}, g, \beta\right)$ the signed count of its points.

As before, this count can change as the metric and perturbation vary, so we need to define a suitable index-theoretic correction term $w\left(X, \mathfrak{s}_{X}, g, \beta\right)$. To do this, choose a 3-manifold $Y \subset X$ representing $1 \in$ $H_{3}(X ; \mathbb{Z})$, and let $\mathfrak{s}_{Y}$ be the restriction of $\mathfrak{s}_{X}$ to $Y$. If $b_{-}^{2}(X)>1$, then we assume as in (26) that $Y$ is an integral homology sphere. Also choose a smooth oriented compact $\operatorname{spin}^{c}$ manifold $Z$ with $\partial\left(Z, \mathfrak{s}_{Z}\right)=\left(Y, \mathfrak{s}_{Y}\right)$. Note that a simply connected $Z$ with $\partial Z=Y$ will have a spin ${ }^{c}$ structure extending $\mathfrak{s}_{Y}$, so there are many choices for $Z$. As in Section 3.1, form the end-periodic $\operatorname{spin}^{c}$ manifold $Z_{+}=Z \cup \tilde{X}_{+}$and extend a regular pair $(g, \beta)$ to an end-periodic pair $(g, \beta)$ on $Z_{+}$. Use the spin ${ }^{c}$ structure $\mathfrak{s}_{Z}$ to lift $\mathfrak{s}_{X}$ to an end-periodic $\operatorname{spin}^{c}$ structure on $Z_{+}$, called $\mathfrak{s}_{Z_{+}}$. As in Theorem 3.1, the $L^{2}$-closure of the operator $D^{+}\left(Z_{+}, \mathfrak{s}_{Z_{+}}, g\right)+\beta$ will be Fredholm. Define the correction term to be

$$
w\left(X, \mathfrak{s}_{X}, g, \beta\right)=\operatorname{ind}_{\mathbb{C}}\left(D^{+}\left(Z_{+}, \mathfrak{s}_{Z_{+}}, g\right)+\beta\right)+\frac{1}{8}\left(\operatorname{sign}(Z)-c_{1}\left(\mathfrak{s}_{Z}\right)^{2}\right) .
$$

The following proposition is an analogue of Proposition 3.2. 
Proposition 10.1. Given either (26) or (27), the correction term $w\left(X, \mathfrak{s}_{X}, g, \beta\right)$ is independent of the choice of $Y \subset X$ and $Z$, and of the way $g, \beta$, and the $\operatorname{spin}^{c}$ structure $\mathfrak{s}_{X}$ are extended over $Z_{+}$.

Given Proposition 10.1, the argument that proves Theorem A can be used essentially word for word to prove the following result.

Theorem 10.2. Let $X$ be a smooth oriented homology oriented 4manifold satisfying conditions (26) or (27), and $\mathfrak{s}_{X}$ a $\operatorname{spin}^{c}$ structure satisfying (28). Then $\lambda_{\mathrm{SW}}\left(X, \mathfrak{s}_{X}\right)=\# \mathcal{M}\left(X, \mathfrak{s}_{X}, g, \beta\right)-w\left(X, \mathfrak{s}_{X}, g, \beta\right)$ is independent of the choice of metric $g$ and generic perturbation $\beta$.

Proof of Proposition 10.1 assuming (26). Given two choices, $Z$ and $Z^{\prime}$ with $\partial Z=\partial Z^{\prime}=Y$, we use the excision principle and the index theorem to obtain

$$
\begin{aligned}
\operatorname{ind}_{\mathbb{C}} D^{+}\left(Z_{+}^{\prime}, \mathfrak{s}_{Z_{+}^{\prime}}, g, \beta\right)-\operatorname{ind}_{\mathbb{C}} D^{+}\left(Z_{+}, \mathfrak{s}_{Z_{+}}, g, \beta\right) \\
=\operatorname{ind}_{\mathbb{C}} D^{+}\left(-Z \cup Z^{\prime}, \mathfrak{s}_{Z} \cup \mathfrak{s}_{Z^{\prime}}\right) \\
=\frac{1}{8}\left(c_{1}\left(\mathfrak{s}_{Z} \cup \mathfrak{s}_{Z^{\prime}}\right)^{2}-\operatorname{sign}\left(-Z \cup Z^{\prime}\right)\right) \\
=\frac{1}{8}\left(\operatorname{sign}(Z)-c_{1}\left(\mathfrak{s}_{Z}\right)^{2}\right)-\frac{1}{8}\left(\operatorname{sign}\left(Z^{\prime}\right)-c_{1}\left(\mathfrak{s}_{Z^{\prime}}\right)^{2}\right),
\end{aligned}
$$

which proves that $w\left(X, \mathfrak{s}_{X}, g, \beta\right)$ is independent of the choices of $Z$ and the extensions.

Let $Y$ and $Y^{\prime}$ be two integral homology spheres carrying the generator of $H_{3}(X ; \mathbb{Z})$. Suppose first that $Y^{\prime}$ is disjoint from $Y$, and write $X=$ $U \cup U^{\prime}$ with $-Y \cup Y^{\prime}=\partial U$. Let $\mathfrak{s}_{U}$ and $\mathfrak{s}_{U^{\prime}}$ denote the restriction of $\mathfrak{s}_{X}$ to $U$ and $U^{\prime}$. To use the excision principle as in the proof of Proposition 3.2, we need to show that

$$
c_{1}\left(\mathfrak{s}_{U}\right)^{2}=\operatorname{sign}(U) \quad \text { and } \quad c_{1}\left(\mathfrak{s}_{U^{\prime}}\right)^{2}=\operatorname{sign}\left(U^{\prime}\right) .
$$

We know that $H^{2}(X)=H^{2}(U) \oplus H^{2}\left(U^{\prime}\right)$, and that the intersection form on each summand is diagonalizable. Therefore, $-c_{1}\left(\mathfrak{s}_{U}\right)^{2} \geq b_{2}(U)$ and $-c_{1}\left(\mathfrak{s}_{U^{\prime}}\right)^{2} \geq b_{2}\left(U^{\prime}\right)$. On the other hand, $c_{1}\left(\mathfrak{s}_{X}\right)^{2}=c_{1}\left(\mathfrak{s}_{U}\right)^{2}+$ $c_{1}\left(\mathfrak{s}_{U^{\prime}}\right)^{2}$, and (29) follows.

If $Y$ and $Y^{\prime}$ are not disjoint, choose lifts of $Y$ and $Y^{\prime}$ to $\tilde{X}$. Translate $Y^{\prime}$ by a sufficiently high prime power $p$ of the covering translation to make it disjoint from $Y$. Then $Y$ and $Y^{\prime}$ cobound a submanifold $U$ of $\tilde{X}$ which embeds into the cyclic $p$-fold cover $\hat{X} \rightarrow X$. Since $p$ is prime, it follows from Smith theory (cf. $[\mathbf{1 7}]$ ) that $b_{1}(\hat{X})=1$. Both the signature and Euler characteristic multiply by $p$ when passing to a cyclic $p$-fold cover; hence $\chi(\hat{X})=p n$ and $\operatorname{sign}(\hat{X})=-p n$ and, in particular, $\hat{X}$ is negative definite. The argument above (with $H^{2}$ replaced by $H^{2}$ /torsion) now shows that (29) holds.

q.e.d. 
Proof of Proposition 10.1 assuming (27). As in the previous proof, we start with disjoint manifolds $Y$ and $Y^{\prime}$, separating $X$ into $U$ and $U^{\prime}$. When $Y$ and $Y^{\prime}$ have nontrivial homology, the relation between the intersection form of $X$ and those of $U$ and $U^{\prime}$ is best understood via Novikov additivity. Following the proof of [4, proposition 7.1], define $A \subset H^{2}(X ; \mathbb{Q})$ as the image of the composition

$$
H^{2}(U, \partial U ; \mathbb{Q}) \rightarrow H^{2}\left(X, U^{\prime} ; \mathbb{Q}\right) \rightarrow H^{2}(X ; \mathbb{Q}),
$$

and define $A^{\prime} \subset H^{2}(X ; \mathbb{Q})$ similarly. The subspaces $A$ and $A^{\prime}$ are mutual annihilators for the intersection form on $X$. Since $H^{2}(X ; \mathbb{Q})=\mathbb{Q}$, at least one of these subspaces must vanish; in particular, $A \cap A^{\prime}=0$. It then follows as in $[4]$ that

$$
\begin{aligned}
H^{2}(X ; \mathbb{Q})=\operatorname{im}\left[H^{2}(U, \partial U ; \mathbb{Q})\right. & \left.\rightarrow H^{2}(U ; \mathbb{Q})\right] \\
& \oplus \operatorname{im}\left[H^{2}\left(U^{\prime}, \partial U^{\prime} ; \mathbb{Q}\right) \rightarrow H^{2}\left(U^{\prime} ; \mathbb{Q}\right)\right],
\end{aligned}
$$

where exactly one of the summands (say the former) is non-zero. The projections onto the summands are given by restriction, hence $c_{1}\left(\mathfrak{s}_{U}\right) \in$ $\operatorname{im}\left[H^{2}(U, \partial U ; \mathbb{Q}) \rightarrow H^{2}(U ; \mathbb{Q})\right]$, and similarly for $c_{1}\left(\mathfrak{s}_{U^{\prime}}\right)$. The latter of course vanishes because it lives in the trivial vector space. As for the former, $c_{1}\left(\mathfrak{s}_{U}\right)^{2} \in \mathbb{Q}$ is well-defined and, as claimed,

$$
c_{1}\left(\mathfrak{s}_{U}\right)^{2}=c_{1}\left(\mathfrak{s}_{X}\right)^{2}=-1=\operatorname{sign}(U) .
$$

If $Y$ and $Y^{\prime}$ intersect, then the argument above which allows to separate them by passing to a covering space will not work, because the condition $b_{2}=1$ need not hold in the covering space. Instead, we appeal to the following principle (cf. $[\mathbf{2 4}, \mathbf{4 1}])$ : There exists a sequence of connected submanifolds $Y=Y_{0}, \ldots, Y_{n}=Y^{\prime}$ such that each $Y_{i}$ carries the generator of $H_{3}(X)$, and $Y_{i+1}$ is disjoint from $Y_{i}$. Thus, the invariants defined by cutting along each $Y_{i}$ in turn are equal, and the independence is proved.

q.e.d.

Note that the argument at the end of the second proof would not work in the situation when $b_{2}>1$, because there is no guarantee that the $Y_{i}$ would be homology spheres.

\section{Examples}

In this section, we discuss the invariant $\lambda_{\mathrm{SW}}(X)$ for mapping tori $X$ of finite order diffeomorphisms $\tau: Y \rightarrow Y$. We succeed in calculating $\lambda_{\mathrm{SW}}(X)$ explicitly for Seifert fibered homology spheres $Y$ and a certain natural $\tau$ associated with $Y$ as a link of a singularity.

11.1. Mapping tori. Let $Y$ be a closed oriented 3-manifold and $\tau$ : $Y \rightarrow Y$ a finite order orientation preserving diffeomorphism such that the mapping torus $X=([0,1] \times Y) /(0, x) \sim(1, \tau(x))$ is a homology $S^{1} \times S^{3}$. Suppose that $Y$ is equipped with a Riemannian metric $g$ such 
that $\tau$ is an isometry; then the product metric on $[0,1] \times Y$ gives rise to a natural metric on $X$ which we call again $g$.

Proposition 11.1. The infinite cyclic cover $\tilde{X}$ of $X$ is isometric to $\mathbb{R} \times Y$.

Proof. The mapping torus $X$ can be written as $X=(\mathbb{R} \times Y) /(t, x) \sim$ $(t+1, \tau(x))$, where the map $(t, x) \rightarrow(t+1, \tau(x))$ is an isometry of the product metric.

q.e.d.

We conclude that a periodic end modeled on the mapping torus of a finite order isometry is isometric to a product end. In particular, the Dirac operator $D^{+}\left(Z_{+}, g\right)$ is isomorphic over the end to $d / d t+D$, where $D$ is the self-adjoint Dirac operator on $Y$. According to [29], the operator $D$ is invertible for a generic metric $g$ on $Y$, and hence the $L^{2}$-closure of $D^{+}\left(Z_{+}, g\right)$ is Fredholm. Therefore,

$$
\begin{aligned}
& w(X, g, 0)=w\left(S^{1} \times Y, g, 0\right) \\
& \quad=\operatorname{ind}_{\mathbb{C}} D^{+}\left(Z_{+}, g\right)+\frac{1}{8} \operatorname{sign}(Z)=-\left(\frac{1}{2} \eta_{\operatorname{Dir}}(Y)+\frac{1}{8} \eta_{\operatorname{Sign}}(Y)\right)
\end{aligned}
$$

by the Atiyah-Patodi-Singer theorem [5], where $\eta_{\text {Dir }}(Y)$ and $\eta_{\text {Sign }}(Y)$ refer to the $\eta$-invariants of, respectively, the Dirac operator $D$ and the odd signature operator on $Y$.

This takes care of the correction term $w(X, g, 0)$. The other ingredient in calculating $\lambda_{\mathrm{SW}}(X)$ is the signed count of points in the moduli space $\mathcal{M}(X, g, 0)$. Since $X$ admits a fixed point free action of $S^{1}$ (which makes it into a circle bundle over the orbifold $Y / \tau)$, we can employ techniques of [6] to identify $\mathcal{M}(X, g, 0)$, for a generic metric $g$, with $\mathcal{M}^{\tau}(Y, g)$, the equivariant Seiberg-Witten moduli space on the 3-manifold $Y$.

Note that using the above approach to computing $\lambda_{\mathrm{SW}}(X)$ for mapping tori is in general problematic because of the equivariant transversality required from the metric $g$. However, this approach works in at least a couple of instances. One is when $\tau$ is the identity so that $X=S^{1} \times Y$ is the product, and the other is when $Y$ is a Seifert fibered homology sphere and $\tau$ is a certain involution associated with $Y$ as a link of a singularity. These two classes of examples will be studied in the rest of this section.

11.2. The product case. Let $X=S^{1} \times Y$ have the product metric. It follows from $\operatorname{Lim}[\mathbf{2 5}]$ that, for a generic metric $g$ on $Y$, we have

$$
\lambda_{\mathrm{SW}}\left(S^{1} \times Y\right)=-\lambda(Y),
$$

where $\lambda(Y)$ is the Casson invariant [2]. This supports the conjecture stated in the introduction because we know that $\lambda_{\mathrm{FO}}\left(S^{1} \times Y\right)=\lambda(Y)$; see $[45]$. 
Remark 11.2. Our orientation convention is that $\lambda(\Sigma(2,3,5))=$ -1 . Since the metric $g$ on $\Sigma(2,3,5)$ has positive scalar curvature, $\mathcal{M}(\Sigma(2,3,5), g)$ is empty. On the other hand, if we choose $Z$ to be the plumbed manifold with the (negative definite) intersection form $E_{8}$, then $\operatorname{sign}(Z) / 8=-1$ and ind $D^{+}\left(Z_{+}, g\right)=0$ (the latter can be found in $[\mathbf{1 5}$, proposition 8]). This fixes the sign in formula (30).

11.3. Seifert fibered homology spheres. Given pairwise relatively prime integers $a_{1}, \ldots, a_{n} \geq 1$, consider the Seifert fibered homology sphere $Y=\Sigma\left(a_{1}, \ldots, a_{n}\right)$. This is an integral homology sphere which will be viewed as a link of a Brieskorn-Hamm complete intersection singularity with real coefficients. It is canonically oriented and admits a fixed point free circle action. This action makes $Y$ into an orbifold circle bundle $\pi: Y \rightarrow F$, where $F$ is the 2-sphere with $n$ singular points of multiplicities $a_{1}, \ldots, a_{n}$. The orbifold Euler characteristic of $F$ is given by the formula

$$
\chi(F)=2-\sum\left(1-1 / a_{k}\right) .
$$

Let $i \eta$ denote the connection form of the circle bundle and $g_{F}$ an orbifold metric on $F$ with constant curvature. We follow $[\mathbf{3 3}]$ and endow $Y$ with the metric $g=\eta^{2}+\pi^{*}\left(g_{F}\right)$ and the connection ${ }^{\circ} \nabla$ on $T Y$ canonically induced by the Levi-Civita connection on $F$. Note that ${ }^{\circ} \nabla$ differs from the standard Levi-Civita connection on $Y$ used in the definition of the Seiberg-Witten invariants.

According to [35, section 2.3], the metric $g$ on $Y$ is generic in that ker $D(Y, g)=0$, and all irreducible solutions are $S^{1-i n v a r i a n t}$ up to gauge transformation. This implies that the moduli space $\mathcal{M}(Y, g)$ of irreducible solutions to the Seiberg-Witten equations on $Y$ with respect to the metric $g$ and the connection ${ }^{\circ} \nabla$ can be identified via pullback with two copies of the space of effective orbifold divisors over $F$ with orbifold degree not exceeding $-\chi(F) / 2$. More precisely, $\mathcal{M}(Y, g)$ contains finitely many components $\mathcal{C}^{+}(\varepsilon)$ and an equal number of components $\mathcal{C}^{-}(\varepsilon)$, both labeled by the vectors $\varepsilon=\left(\varepsilon_{1}, \ldots, \varepsilon_{n}\right)$ such that $0 \leq \varepsilon_{k}<a_{k}$ and

$$
\sum \varepsilon_{k} / a_{k} \leq-\chi(F) / 2 .
$$

The components $\mathcal{C}^{ \pm}(\varepsilon)$ consist of holomorphic (respectively, anti-holomorphic) vortices on $F$. If $n=3$ or $n=4$, each of the components $\mathcal{C}^{ \pm}(\varepsilon)$ is just a point.

Regarding the Seiberg-Witten moduli space $\mathcal{M}(Y, g)$ corresponding to the metric $g$ and the Levi-Civita connection, Nicolaescu [36, theorem 3.1] showed that there is a natural bijective correspondence between $\mathcal{M}(Y, g)$ and $\mathcal{M}(Y, g)$ for all metrics $g$ on $Y$ as above with sufficiently short circle fibers.

Let $\tau: Y \rightarrow Y$ be induced by the complex conjugation on the link $Y$. Then $\tau$ is an involution that makes $Y$ into a double branched cover 
of $S^{3}$ with branch set a Montesinos knot. It commutes with the projection $\pi: Y \rightarrow F$ and thus defines an anti-holomorphic involution on $F$. In particular, it interchanges the holomorphic and anti-holomorphic vortices $\mathcal{C}^{ \pm}(\varepsilon)$ on $F$ so that ${ }^{\circ} \mathcal{M}^{\tau}(Y, g)$ and hence $\mathcal{M}^{\tau}(Y, g)$ are empty. Therefore, for the mapping torus $X$ of $\tau$,

$$
\lambda_{\mathrm{SW}}(X)=\frac{1}{2} \eta_{\text {Dir }}(Y)+\frac{1}{8} \eta_{\mathrm{Sign}}(Y) .
$$

The latter can be calculated explicitly in terms of either integral lattice points or Dedekind sums; see [36]. On the other hand, $\lambda_{\mathrm{FO}}(X)$ is equal to the equivariant Casson invariant $\lambda^{\tau}(Y)$, also known as the $\bar{\mu}^{-}$ invariant of Neumann and Siebenmann; see for instance $[\mathbf{1 0}]$ or $[\mathbf{4 7}]$. Our conjecture is then equivalent to showing that

$$
\frac{1}{2} \eta_{\text {Dir }}(Y)+\frac{1}{8} \eta_{\text {Sign }}(Y)=-\bar{\mu}(Y)
$$

for all Seifert fibered homology spheres $Y=\Sigma\left(a_{1}, \ldots, a_{n}\right)$. We succeeded in checking that (31) is true by a direct calculation with Dedekind sums; a complete proof can be found in $[\mathbf{4 6}]$.

\section{Appendix A. Existence of special paths}

In this appendix, we verify that any regular path of metrics and perturbations can be homotoped rel endpoints to a path $\left(g_{I}, \beta_{I}\right)$ so that near any points $t$ for which $\mathcal{M}^{0}\left(X, g_{t}, \beta_{t}\right)$ is non-empty, the metric is constant. This was stated as Theorem 2.5 in Section 2.3.

Let us write $\mathcal{R}$ for the space of Riemannian metrics on $X$, with the $C^{k}$ topology for some sufficiently large $k$. As in Section 2.2, form the space $\widetilde{\mathcal{Z}}_{\mathcal{R}} \subset \mathcal{R} \times \widetilde{\mathcal{B}}$ consisting of quadruples $(g, A, s, \varphi)$ with $D_{A}^{+}(X, g)(\varphi)=0$. The proof of lemma 27.1.1 in [23], which shows that $\widetilde{\mathcal{Z}}$ is a Hilbert submanifold of $\widetilde{\mathcal{B}}$ for any metric $g$, goes through with little change to show that $\widetilde{\mathcal{Z}}_{\mathcal{R}}$ is a Hilbert submanifold of $\mathcal{R} \times \widetilde{\mathcal{B}}$. The equation $s=0$ defines a codimension one submanifold $\partial \mathcal{Z}_{\mathcal{R}} \subset \widetilde{\mathcal{Z}}_{\mathcal{R}}$. The projection $(g, A, s, \varphi) \rightarrow g$ induces submersions $\widetilde{\mathcal{Z}}_{\mathcal{R}} \rightarrow \mathcal{R}$ and $\partial \mathcal{Z}_{\mathcal{R}} \rightarrow \mathcal{R}$.

Let $\Omega_{\mathcal{R}}$ be the subspace of $\mathcal{R} \times \Omega^{2}(X, i \mathbb{R})$ comprising the pairs $(g, \omega)$ such that $\omega$ is self-dual with respect to the metric $g$. Projection onto the first factor makes it into a Hilbert bundle $\pi: \Omega_{\mathcal{R}} \rightarrow \mathcal{R}$. As such, it is isomorphic to the parameterized perturbation space $\mathcal{P}_{\mathcal{R}}$, which is defined as the Hilbert bundle over $\mathcal{R}$ comprising the pairs $(g, \beta) \in$ $\mathcal{R} \times \Omega^{1}(X, i \mathbb{R})$ such that $d^{*} \beta=0$ and $\beta \in \mathcal{H}^{1}(X, i \mathbb{R})^{\perp}$ with respect to $g$. The bundle isomorphism sends $(g, \beta) \in \mathcal{P}_{\mathcal{R}}$ to $\left(g, d^{+g} \beta\right) \in \Omega_{\mathcal{R}}$. 
The above maps can be included into the commutative diagram

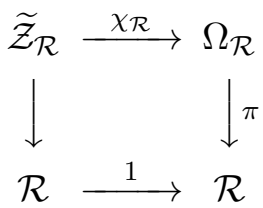

where the map $\chi_{\mathcal{R}}$ is given by $\chi_{\mathcal{R}}(g, A, s, \varphi)=\left(g, F_{A}^{+g}-s^{2} \tau_{g}(\varphi)\right)$. The restriction of $\chi_{\mathcal{R}}$ to $\partial \mathcal{Z}_{\mathcal{R}}$ will be denoted $\partial \chi_{\mathcal{R}}$. Note that the commutative diagram (2) is just the pullback of the diagram (32) via the map $g_{I}: I \rightarrow \mathcal{R}$.

It follows from Theorem 2.4 that a generic path $\gamma: I \rightarrow \mathcal{P}_{\mathcal{R}}$ connecting regular values $\gamma_{0}=\left(g_{0}, \beta_{0}\right)$ and $\gamma_{1}=\left(g_{1}, \beta_{1}\right)$ of $\chi_{\mathcal{R}}$ has the property that the composite path $I \rightarrow \mathcal{P}_{\mathcal{R}} \rightarrow \Omega_{\mathcal{R}}$, which we will also call $\gamma$, is transverse to both $\partial \chi_{\mathcal{R}}$ and $\chi_{\mathcal{R}}$. We will say that $\gamma$ is vertical near its intersection point with $\operatorname{im}\left(\partial \chi_{\mathcal{R}}\right)$ if its metric component is constant in a neighborhood of that point.

Theorem A.1. Any generic path $\gamma: I \rightarrow \mathcal{P}_{\mathcal{R}}$ as above can be homotoped rel its endpoints to a generic path that is vertical near every intersection point with $\operatorname{im}\left(\partial \chi_{\mathcal{R}}\right)$.

This theorem is a re-statement of Theorem 2.5. Its proof will have two steps: one local, and a second involving some global arguments. The first step is contained in the following three lemmas, all of which are concerned with a point $z \in \partial \mathcal{Z}_{\mathcal{R}}$ such that $\left(\partial \chi_{\mathcal{R}}\right)(z)=\gamma_{0}=(g, w) \in$ $\Omega_{\mathcal{R}}$, after a proper reparameterization of $I$.

Lemma A.2. Suppose that $\gamma:[-1,1] \rightarrow \mathcal{P}_{\mathcal{R}}$ is a generic path, and let $z \in \partial \mathcal{Z}_{\mathcal{R}}$ be such that $\chi_{\mathcal{R}}(z)=\gamma_{0}$. Then the differential at $z$ of the map $\partial \chi_{\mathcal{R}}: \partial \mathcal{Z}_{\mathcal{R}} \rightarrow \Omega_{\mathcal{R}}$ is injective.

Proof. In our situation, the map $\chi_{\mathcal{R}}$ has index zero, and its restriction $\partial \chi_{\mathcal{R}}$ has index -1 . Because of transversality to $\gamma$, the differential at $z$ of the map $\partial \chi_{\mathcal{R}}$ has image of codimension one; hence it must be injective.

q.e.d.

By the inverse function theorem, there is a neighborhood $U$ of $z$ in $\partial \mathcal{Z}_{\mathcal{R}}$ such that the restriction of $\partial \chi_{\mathcal{R}}$ to $U$ is an embedding into an open ball $W \subset \Omega_{\mathcal{R}}$. Let us write $V=\left(\partial \chi_{\mathcal{R}}\right)(U)$.

Lemma A.3. There is a unique unit vector $\nu \in T_{w} \Omega_{\mathcal{R}}$ such that

1) $\nu$ is vertical, that is, $\nu \in \operatorname{ker}\left(D_{w} \pi\right)$ with $\pi: \Omega_{\mathcal{R}} \rightarrow \mathcal{R}$,

2) $\mathbb{R} \cdot \nu \oplus T_{w} V=T_{w} \Omega_{\mathcal{R}}$, and

3) $\nu=a \gamma^{\prime}(0)+Y$ for some $Y \in T_{w} V$ and $a>0$.

Proof. Since $\partial \mathcal{Z}_{\mathcal{R}} \rightarrow \mathcal{R}$ is a submersion, the commutativity of the diagram (32) implies that the differential at $w$ of $\pi: \Omega_{\mathcal{R}} \rightarrow \mathcal{R}$ is surjective 
when restricted to $T_{w} V \subset T_{w} \Omega_{\mathcal{R}}$. Since $V$ has codimension one in $\Omega_{\mathcal{R}}$, the result follows.

q.e.d.

In the proof of Proposition A.5 below, we will need an estimate saying that the Seiberg-Witten map $\partial \chi_{\mathcal{R}}$ is close to its derivative.

Lemma A.4. There is a constant $C$ such that

$$
\left\|\left(\partial \chi_{\mathcal{R}}\right)\left(z_{1}\right)-D_{z}\left(\partial \chi_{\mathcal{R}}\right)\left(z_{1}-z\right)\right\| \leq C\left\|z_{1}-z\right\|^{2}
$$

for $\left\|z_{1}-z\right\|$ sufficiently small. In this inequality, the distances are computed in $\mathcal{R} \times \widetilde{\mathcal{B}}$ and $\mathcal{R} \times \Omega^{2}(X, i \mathbb{R})$, respectively.

Proof. According to Taylor's theorem for smooth functions on Banach spaces (cf. [53, §4.5]), an estimate of the form (33) holds whenever the second derivative of $\partial \chi_{\mathcal{R}}$ is uniformly bounded in a ball around $z$. The map $\partial \chi_{\mathcal{R}}$ is linear in the connection $A$ (and independent of $\varphi$ since $z \in \partial \mathcal{Z}_{\mathcal{R}}$ ), so the only issue is the dependence on the metric $g$. Trivialize the bundle $\Omega_{\mathcal{R}} \rightarrow \mathcal{R}$ using the maps $b_{t}$ as in Remark 2.3, and observe that $\partial \chi_{\mathcal{R}}$ only depends on $g$ via the Hodge star operator. Simple linear algebra shows that this dependence is quartic, from which the bound follows.

q.e.d.

A similar estimate, $\left\|\gamma(t)-t \gamma^{\prime}(0)\right\| \leq C t^{2}$, holds for the path $\gamma$. These three lemmas yield the local statement we need.

Proposition A.5. Suppose that $\gamma:[-1,1] \rightarrow \Omega_{\mathcal{R}}$ is a generic path in $W$ whose pre-image in $U$ is a single point $z$ with $w=\left(\partial \chi_{\mathcal{R}}\right)(z)=\gamma_{0}$. For any sufficiently small $\delta>0$, there is a generic path $\gamma_{\delta}$ such that $\gamma_{\delta} \cap V=\gamma \cap V$ and $\gamma_{\delta}$ crosses $V$ vertically at $w$. In addition, the two paths are homotopic by a homotopy supported in $(-\delta, \delta)$.

Proof. By choosing local coordinates in the ball $W$ near $w$, we can assume that $V \subset W$ is a linear ball of codimension one. In particular, $V$ separates $W$ into two components, and likewise the intersection $V \cap \partial W$ separates $\partial W$ into two components. Since $\gamma$ is transversal to $V$ at $w$, it must cross from one side of $V$ to the other. Choosing $W$ small enough, we can assume that

1) $\gamma^{-1}(W)$ is a single interval $(-\delta, \delta)$ for some $\delta>0$,

2) a small positive multiple $a_{+} \nu$ of the vector $\nu$ from Lemma A.3 lies on the same side of $V$ as $\gamma(t)$ for $t \in(0, \delta)$,

3) a small negative multiple $a_{-} \nu$ lies on the same side of $V$ as $\gamma(t)$ for $t \in(-\delta, 0)$, and

4) the multiples $a_{ \pm} \nu$ belong to $\partial W$.

Now we can construct a path $\gamma_{\delta}$ as follows. It is equal to $\gamma$ outside of $(-\delta, \delta)$. From $-\delta$ to $-\delta / 2$, it follows a path in $\partial W$ (avoiding $V \cap \partial W$ ) from $\gamma(-\delta)$ to $a_{-} \nu$. From $-\delta / 2$ to $\delta / 2$, it follows the vertical line from $a_{-} \nu$ to $a_{+} \nu$. Finally, from $\delta / 2$ to $\delta$, it follows a path in $\partial W$ (avoiding $V \cap \partial W)$ from $a_{+} \nu$ to $\gamma(\delta)$. See Figure 4 .

q.e.d. 


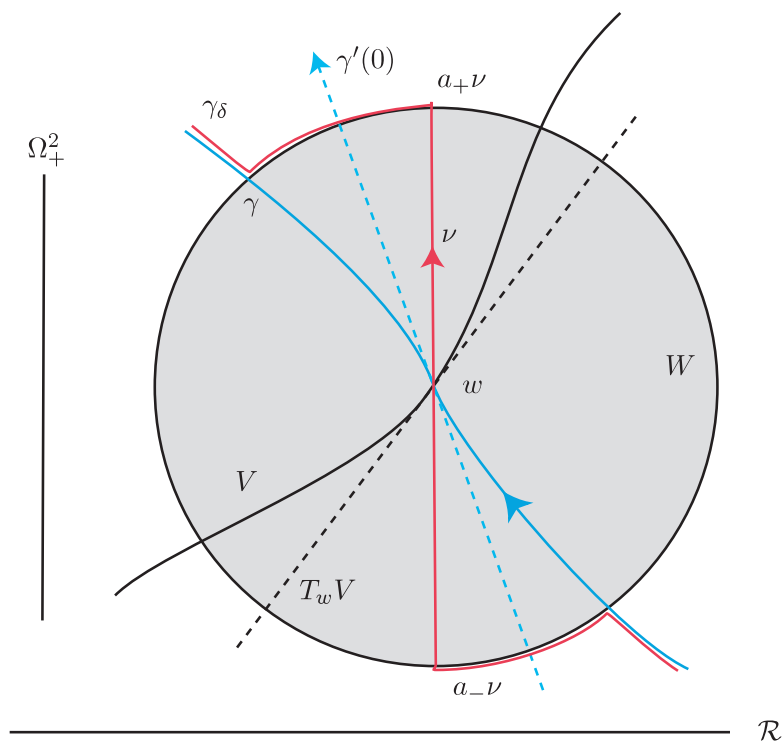

Figure 4. Local modification of $\gamma$

The main issue in deriving Theorem A.1 from the above local result, which is Proposition A.5, is that a given intersection point $w \in$ $\operatorname{im}\left(\partial \chi_{\mathcal{R}}\right) \cap \gamma$ may have several pre-images in $\partial \mathcal{Z}_{\mathcal{R}}$. There are at most finitely many such pre-images, which follows from the map $\chi_{\mathcal{R}}$ being proper, as proved in [23, theorem 5.2.1].

Denote the points in $\left(\partial \chi_{\mathcal{R}}\right)^{-1}(w)$ by $z_{k} \in \partial \mathcal{Z}_{\mathcal{R}}, k=1, \ldots, n$. Choose an open ball $W$ centered at $w$ with the property that, for each $k$, the point $z_{k}$ has a neighborhood $U_{k} \subset \partial \mathcal{Z}_{\mathcal{R}}$ such that the restriction of $\partial \chi_{\mathcal{R}}$ to $U_{k}$ is an embedding into $W$. Note that the sheets $V_{k}=\left(\partial \chi_{\mathcal{R}}\right)\left(U_{k}\right)$ meeting at $w$ are all embedded, but they might be transverse or tangent to each other.

Lemma A.6. If the ball $W$ as above is chosen sufficiently small, then

$$
\left(\partial \chi_{\mathcal{R}}\right)^{-1}(W) \subset \bigsqcup_{k} U_{k}
$$

Proof. Suppose to the contrary that there is no such $W$. Then there is a sequence of points $u_{i} \in \partial \mathcal{Z}_{\mathcal{R}}$ away from the $U_{k}$ with bounded energy (again, referring to $\left[\mathbf{2 3}\right.$, theorem 5.2.1]) and with $\left(\partial \chi_{\mathcal{R}}\right)\left(u_{i}\right)$ converging to $w$. It follows that a subsequence of the $u_{i}$ converges in $\partial \mathcal{Z}_{\mathcal{R}}$, and then of course it must converge to one of the $z_{k}$. This contradiction establishes the claim.

q.e.d.

Next, we claim that we may assume that all of the $V_{k}$ are tangent at their common intersection point $w$. Suppose that $T_{w} V_{i} \neq T_{w} V_{j}$ for 
some $i \neq j$. Since both of these subspaces have codimension one (cf. the proof of Lemma A.2), they must in fact be transverse. It follows that the intersection $V_{i} \cap V_{j}$ must be a codimension 2 submanifold near $w$. A small perturbation of $\gamma$ will then suffice to avoid all such double point sets.

Finally, we are in the situation where at any intersection point $w$, all of the finitely many $V_{k}$ that meet $W$ are tangent. It follows that the vector $\nu$ constructed in Lemma A.3 lies on the same side of all of the $V_{k}$, and so (if $W$ is sufficiently small) the curve constructed by the local deformation in Proposition A.5 meets all sheets vertically. Since the deformation takes place in $W$, no new intersections have been introduced, and Theorem A.1 is proved.

\section{References}

[1] S. Agmon \& L. Nirenberg, Properties of solutions of ordinary differential equations in Banach space, Comm. Pure Appl. Math. 16 (1963) 121-239, MR 0155203, Zbl 0117.10001.

[2] S. Akbulut \& J. McCarthy, Casson's invariant for oriented homology 3-spheres. An exposition. Mathematical Notes, 36. Princeton University Press, Princeton, 1990, MR 1030042, Zbl 0695.57011.

[3] M. Atiyah \& I. Singer, The index of elliptic operators. I, Ann. of Math. (2) 87 (1968) 484-530, MR 0236950, Zbl 0164.24001.

[4] — The index of elliptic operators. III, Ann. of Math. (2) 87 (1968) 546-604, MR 0236952, Zbl 0164.24301

[5] M. Atiyah, V. Patodi \& I. Singer, Spectral asymmetry and Riemannian geometry. I, Math. Proc. Cambridge Philos. Soc. 77 (1975) 43-69, MR 0397797, Zbl 0297.58008.

[6] S. Baldridge, Seiberg-Witten invariants, orbifolds, and circle actions, Trans. Amer. Math. Soc. 355 (2003) 1669-1697, MR 1946410, Zbl 1013.57019.

[7] B. Booß-Bavnbek \& K. Wojciechowski, Elliptic boundary problems for Dirac operators, Birkhäuser, 1993, MR 1233386, Zbl 0797.58004.

[8] B. Charbonneau, Analytic aspects of periodic instantons. Ph.D. thesis, MIT 2004. http://hdl.handle.net/1721.1/26746

[9] W. Chen, Casson's invariant and Seiberg-Witten gauge theory, Turkish J. Math. 21 (1997) 61-81, MR 1456160, Zbl 0891.57021.

[10] O. Collin \& N. Saveliev, Equivariant Casson invariants via gauge theory, J. Reine Angew. Math. 541 (2001) 143-169, MR 1876288, Zbl 0989.57013.

[11] S. Donaldson \& P. Kronheimer, The Geometry of Four-Manifolds, The Clarendon Press, Oxford University Press, New York, 1990, MR 1079726, Zbl 0904.57001 .

[12] S. K. Donaldson, The Seiberg-Witten equations and 4-manifold topology, Bull. Amer. Math. Soc. (N.S.) 33 (1996) 45-70, MR 1339810, Zbl 0872.57023.

[13] J. Eells \& L. Lemaire, A report on harmonic maps, Bull Lond. Math. Soc. 10 (1978) 1-68, MR 0495450, Zbl 0401.58003.

[14] K. Frøyshov, Monopole Floer homology for rational homology 3-spheres, Duke Math. J. 155 (2010), 519-576, MR 2738582, Zbl 05834475 
[15] - The Seiberg-Witten equations and four-manifolds with boundary, Math. Res. Lett. 3 (1996) 373-390, MR 1397685, Zbl 0872.57024.

[16] M. Furuta \& H. Ohta, Differentiable structures on punctured 4-manifolds. Topology Appl. 51 (1993) 291-301, MR 1237394, Zbl 0799.57009.

[17] P. Gilmer, Configurations of surfaces in 4-manifolds, Trans. Amer. Math. Soc. 264 (1981) 353-380, MR 0603768, Zbl 0509.57023.

[18] C. Gordon, Some aspects of classical knot theory. Knot theory (Proc. Sem., Plans-sur-Bex, 1977), pp. 1-60, Lecture Notes in Math. 685, Springer, Berlin, 1978, MR 0521730, Zbl 0386.57002.

[19] M. Gromov \& H. B. Lawson, Jr., Positive scalar curvature and the Dirac operator on complete Riemannian manifolds, Inst. Hautes Études Sci. Publ. Math. No. 58 (1983) 83-196, MR 0720933, Zbl 0538.53047.

[20] E. I. Jury, Theory and Application of the Z-Transform Method, Wiley, New York, 1964.

[21] T. Kato, Perturbation theory for linear operators, second edition, Grundlehren der Mathematischen Wissenschaften, Band 132, Springer-Verlag, Berlin-New York, 1980, MR 1335452, Zbl 0836.47009.

[22] R. Kirby \& L. Siebenmann, Foundational essays on topological manifolds, smoothings, and triangulations, Annals of Math. Studies, No. 88, Princeton University Press, Princeton, 1977, MR 0645390, Zbl 0361.57004.

[23] P. Kronheimer \& T. Mrowka, Monopoles and three-manifolds, Cambridge Univ. Press, Cambridge, 2007, MR 2388043, Zbl 1158.57002.

[24] W. B. R. Lickorish, An introduction to knot theory, Graduate Texts in Mathematics, 175, Springer-Verlag, New York, 1997, MR 1472978, Zbl 0886.57001.

[25] Y. Lim, Seiberg-Witten invariants for 3-manifolds in the case $b_{1}=0$ or 1 , Pacific J. Math. 195 (2000) 179-204, MR 1781619, Zbl 1015.57022.

[26] - The equivalence of Seiberg-Witten and Casson invariants for homology 3-spheres, Math. Res. Lett. 6 (1999) 631-643, MR 1739221, Zbl 0948.57007.

[27] A. Lobb \& R. Zentner, On Casson-type instanton moduli spaces over negative definite four-manifolds. Preprint http://arxiv.org/abs/0802.4041v3 (2008).

[28] R. Lockhart \& R. McOwen, Elliptic differential operators on noncompact manifolds, Ann. Scuola Norm. Sup. Pisa Cl. Sci. (4) 12 (1985) 409-447, MR 0837256, Zbl 0615.58048.

[29] S. Maier, Generic metrics and connections on Spin- and Spin ${ }^{c}$-manifolds, Comm. Math. Phys. 188 (1997) 407-437, MR 1471821, Zbl 0899.53036.

[30] R. Mazzeo, D. Pollack, \& K. Uhlenbeck, Moduli spaces of singular Yamabe metrics, J. Amer. Math. Soc. 9 (1996) 303-344, MR 1356375, Zbl 0849.58012.

[31] J. W. Morgan, The Seiberg-Witten equations and applications to the topology of smooth four-manifolds, Mathematical Notes, 44, Princeton University Press, Princeton, 1996, MR 1367507, Zbl 0846.57001.

[32] J. Morgan \& Z. Szabó, Homotopy K3 surfaces and mod 2 Seiberg-Witten invariants, Math. Res. Lett. 4 (1997) 17-21, MR 1432806, Zbl 0876.57031.

[33] T. Mrowka, P. Ozsváth \& B. Yu, Seiberg-Witten monopoles on Seifert fibered spaces, Comm. Anal. Geom. 5 (1997) 685-791, MR 1611061, Zbl 0933.57030.

[34] S. Nazarov, Elliptic boundary value problems with periodic coefficients in a cylinder. (Russian) Izv. Akad. Nauk SSSR Ser. Mat. 45 (1981) 101-112, MR 0607578, Zbl 0483.35032. 
[35] L. Nicolaescu, Eta invariants of Dirac operators on circle bundles over Riemann surfaces and virtual dimensions of finite energy Seiberg-Witten moduli spaces, Israel J. Math. 114 (1999) 61-123, MR 1738674, Zbl 0998.58022.

[36] L. Nicolaescu, Finite energy Seiberg-Witten moduli spaces on 4-manifolds bounding Seifert fibrations, Comm. Anal. Geom. 8 (2000) 1027-1096, MR 1846125, Zbl 1001.58004 .

[37] L. Nicolaescu, Notes on Seiberg-Witten Theory, Graduate Studies in Mathematics, 28, Amer. Math. Soc., Providence, 2000, MR 1787219, Zbl 0978.57027.

[38] E. Ogasa, Ribbon-moves of 2-links preserve the $\mu$-invariant of 2-links, J. Knot Theory Ramifications 13 (2004) 669-687, MR 2080127, Zbl 1082.57017.

[39] C. Okonek \& A. Teleman, Seiberg-Witten invariants for 4-manifolds with $b_{+}=0$. Complex analysis and algebraic geometry, pp. 347-357, de Gruyter, Berlin, 2000, MR 1648803, Zbl 1034.53091.

[40] M. Reed \& B. Simon, Methods of modern mathematical physics. IV. Analysis of operators, Academic Press, New York-London, 1978, MR 0493421, Zbl 0401.47001

[41] P. M. Rice, Equivalence of Alexander matrices, Math. Ann. 193 (1971), 65-75, MR 0287528, Zbl 0205.28001.

[42] D. Ruberman, Doubly slice knots and the Casson-Gordon invariants, Trans. Amer. Math. Soc. 279 (1983) 569-588, MR 0709569, Zbl 0531.57017.

[43] D. Ruberman and N. Saveliev, Casson-type invariants in dimension four. Geometry and Topology of Manifolds, 281-306, Fields Inst. Commun. 47, Amer. Math. Soc., Providence, 2005, MR 2189939, Zbl 1096.57012.

[44] - Dirac operators on manifolds with periodic ends, J. Gökova Geom. Top. GGT 1 (2007) 33-50, MR 2386534, Zbl 1185.58018.

[45] — Rohlin's invariant and gauge theory. II. Mapping tori, Geom. Topol. 8 (2004) 35-76 (electronic), MR 2033479, Zbl 1063.57025.

[46] - The $\bar{\mu}$-invariant of Seifert fibered homology spheres and the Dirac operator, http://arxiv.org/abs/1009.3201. Geom. Dedic. (to appear)

[47] N. Saveliev, Invariants for Homology 3-Spheres, Encyclopaedia of Mathematical Sciences, 140, Springer-Verlag, Berlin, 2002, MR 1941324, Zbl 0998.57001.

[48] M. Scharlemann, Constructing strange manifolds with the dodecahedral space, Duke Math. J. 43 (1976) 33-40, MR 0402760, Zbl 0331.57007.

[49] S. Smale, An infinite dimensional version of Sard's theorem, Amer. J. Math. 87 (1965) 861-866, MR 0185604, Zbl 0143.35301.

[50] C. Taubes, Casson's invariant and gauge theory, J. Differential Geom. 31 (1990) 547-599, MR 1037415, Zbl 0702.53017.

[51] — Gauge theory on asymptotically periodic 4-manifolds, J. Differential Geom. 25 (1987) 363-430, MR 0882829, Zbl 0615.57009.

[52] A. Teleman, Harmonic sections in sphere bundles, normal neighborhoods of reduction loci, and instanton moduli spaces on definite 4-manifolds, Geom. Topol. 11 (2007) 1681-1730, MR 2350464, Zbl 1138.57030.

[53] E. Zeidler, Applied Functional Analysis. Main principles and their applications, Springer-Verlag, New York, 1995, MR 1347692, Zbl 0834.46003.

[54] R. Zentner, A vanishing result for a Casson-type instanton invariant, Preprint http://arxiv.org/abs/0911.2772 (2009). 
Department of Mathematics Massachusetts Institute of Technology CAMBRIDGE MA 02139

E-mail address: mrowka@mit.edu

Department of Mathematics MS 050

BRANDEIS UNIVERSITY

WaLtham, MA 02454

E-mail address: ruberman@brandeis.edu

Department of MATHEMATiCs UNIVERSITY OF MIAMI PO Box 249085 Coral Gables, FL 33124

E-mail address: saveliev@math.miami.edu 